\title{
Die Wirkung der Authentizität von Lernort und Laborgeräten auf das situationale Interesse und die Relevanzwahrnehmung beim Besuch eines naturwissenschaftlichen Schülerlabors
}

\author{
Tobias Schüttler $^{1,2}$ (D) Bianca Watzka' ${ }^{1}$ - Raimund Girwidz ${ }^{1}$ (D) $\cdot$ Bernhard Ertl $^{3}$ (D)
}

Eingegangen: 7. September 2020 / Angenommen: 3. Mai 2021 / Online publiziert: 10. Juni 2021

(c) Der/die Autor(en) 2021

\section{Zusammenfassung}

Als besonderes Merkmal von Schülerlaboren, die u. a. das Interesse an Naturwissenschaften fördern sollen, wird häufig die Authentizität der Lernumgebung genannt. Allerdings besteht Klärungsbedarf, welche Gestaltungsaspekte von authentischen Lernsettings interesseförderlich wirken. Die Faktoren Authentizität des Lernorts und Echtheit der Laborgeräte standen daher bei der hier beschriebenen quasi-experimentellen Interventionsstudie im $2 \times 2$-faktoriellen Design im Vordergrund. Untersucht wurde die Wirkung dieser Faktoren auf das situationale Interesse sowie auf die subjektiv wahrgenommene inhaltliche Relevanz und die wahrgenommene Authentizität der Laborgeräte beim Besuch eines forschungsnahen, naturwissenschaftlichen Schülerlabors. Dazu wurde ein Workshop zur Infrarotfernerkundung entwickelt und mit insgesamt 166 Schüler*innen der zehnten Klasse an unterschiedlich authentischen Lernorten (Schülerlabor vs. Schule) durchgeführt. Kontrastierend kamen an beiden Orten echte High-End-Laborgeräte, die im Allgemeinen nur Forschungseinrichtungen zur Verfügung stehen, bzw. vereinfachte, für Schulen verfügbare, weniger authentische Low-Cost-Geräte zum Einsatz. Die Arbeit mit den echten Laborgeräten führte unabhängig vom Lernort zu einer stärkeren Authentizitätswahrnehmung. Die Wahrnehmung inhaltlicher Relevanz hingegen war unabhängig von den Laborgeräten und wurde am authentischen Lernort signifikant höher bewertet als in der Schule. Das situationale epistemische Interesse war in der Gruppe am größten ausgeprägt, die am authentischen Lernort mit echten Laborgeräten arbeiten konnte.

Schlüsselwörter Interesse $\cdot$ Authentizität · Inhaltliche Relevanz · Infrarotfernerkundung · Schülerlabor

Tobias Schüttler

tobias.schuettler@1mu.de

1 Ludwig-Maximilians-Universität München,

Theresienstraße 37, 80333 München, Deutschland

2 Deutsches Zentrum für Luft- und Raumfahrt, Münchenerstraße 20, 82234 Weßling, Deutschland

3 Universität der Bundeswehr München,

Werner-Heisenberg-Weg 39, 85577 Neubiberg, Deutschland 


\title{
Effects of an Authentic Location and Laboratory Equipment for the Situational Interest and the Perception of Content Relevance when Visiting an Out-of-School Science Lab
}

\begin{abstract}
A special uniqueness often mentioned for Out-of-School Labs, aiming to promote interest in science, is the authenticity of the learning environment. However, there is a lack of research on which design aspects of authentic learning environments have a positive effect on students' interests. The quasi-experimental intervention study in a $2 \times 2$-factorial design presented here examined the effect of an authentic location and laboratory equipment for the situational interest and the perception of content relevance and of the authenticity of the laboratory equipment when visiting an Out-of-School Science Lab. For this, we developed a workshop for tenth graders in the context of infrared remote sensing. The workshop took place at different authentic learning locations (Out-of-School Science Lab vs. school). At both locations a total of 166 students could work with differently authentic equipment: On the one hand, genuine authentic laboratory equipment, which is usually only available to research institutions, and on the other hand, simplified, partly newly developed tools that are available for schools. Working with the real laboratory equipment led to a stronger perception of authenticity regardless of the learning location. The perception of content relevance, on the other hand, was independent of the laboratory equipment and was rated significantly higher at the authentic learning location than at school. The epistemic aspect of the situational interest was most pronounced in the group, which could work with real laboratory equipment at the authentic learning location of the out-of-school lab.
\end{abstract}

Keywords Interest $\cdot$ Authenticity $\cdot$ Content relevance $\cdot$ Infrared remote sensing $\cdot$ Out-of-School Science Lab

\section{Einleitung}

Für das schulische Lernen wird - neben Leistungsmotiven und Zielorientierung - Interesse als eines der wichtigsten dispositionalen Motivationsmerkmale genannt (vgl. Schiefele 2009). Das Interesse von Jugendlichen an Naturwissenschaften, insbesondere den Fächern Physik und Chemie, wird jedoch vielfach als gering beschrieben und nimmt zudem im Laufe der Schulzeit ab (u. a. Schiefele 2009; Krapp und Prenzel 2011; Gebhard et al. 2017). Die Ergebnisse deutscher Schüler*innen bei PISA 2015 untermauern diese Befunde (Reiss et al. 2016). Das Interesse an naturwissenschaftlichen Themen steht in engem Zusammenhang mit naturwissenschaftlichen Karriereerwartungen von Jugendlichen (Taskinen et al. 2013). Da diese auch die tatsächlichen Entscheidungen bezüglich Ausbildungs- und Berufswahl beeinflussen (vgl. Tai et al. 2006), ist das geringe und zudem abnehmende Interesse an naturwissenschaftlichen Fächern also in vielerlei Hinsicht problematisch nicht nur aus der schulischen Perspektive, sondern auch aus gesamtgesellschaftlicher und ökonomischer Sicht eines von Hochtechnologien abhängigen Landes.

Auf Basis der Selbstbestimmungstheorie von Deci und Ryan (2002) können verschiedene Interventionsbereiche zur Interesseförderung abgeleitet werden (vgl. Schiefele 2009). Die Erfüllung der Basic Needs, also Kompetenzwahrnehmung, Selbstbestimmung und soziale Eingebundenheit, wird als Voraussetzung für die Entstehung und Aufrechterhaltung von fachlichen Interessen genannt (Deci und Ryan 2002; Schiefele 2009; Krapp 2005). Daneben spielt aber auch die Bedeutsamkeit des Lerngegenstands eine wichtige Rolle. Eng damit verbunden ist die wahrgenommene inhaltliche Relevanz (Parchmann und Kuhn 2018). Eine Möglichkeit, die Relevanzwahrnehmung und das Interesse bei den Lernenden zu steigern, stellt Unterricht mit Einbeziehung authentischer Kontexte dar (Duit 2010; Kuhn 2010; Watzka und Girwidz 2015; Parchmann und Kuhn 2018; Habig et al. 2018). Einen ähnlichen Ansatz verfolgen Schülerlabore, wobei hier zu einem authentischen, oft aus der echten Forschung stammenden inhaltlichen Kontext, noch ein authentischer Lernort sowie authentische Laborgeräte bzw. Materialien und Arbeitsweisen hinzukommen (Euler und Schüttler 2020). Die förderliche Wirkung von kontextorientiertem Unterricht und von Schülerlaborbesuchen für das Interesse an Naturwissenschaften konnte bereits vielfach belegt werden (vgl. zusammenfassend Bennett et al. 2007; Pawek 2019). Allerdings wird darauf hingewiesen, dass bislang noch $\mathrm{zu}$ wenig systematisch untersucht wurde, welche Faktoren von authentischen Lernumgebungen insbesondere auch nachhaltig interesseförderlich wirken (vgl. Bennett et al. 2007; Demuth et al. 2008; Lewalter und Geyer 2009; van Vorst et al. 2015; Parchmann und Kuhn 2018; ItzekGreulich und Vollmer 2018). Die hier beschriebene Studie untersuchte daher den Einfluss der Authentizität von Lernort und Laborgeräten auf das situationale Interesse sowie auf die Wahrnehmung inhaltlicher Relevanz und der wahrgenommenen Authentizität der verwendeten Laborgeräte beim Besuch eines wissenschaftsnahen, klassischen Schülerlabors. 


\section{Theoretischer Hintergrund}

\section{Interesse}

Das entscheidende Merkmal, welches Interesse von anderen Konstrukten wie intrinsischer Motivation abgrenzt, ist, dass es immer auf einen bestimmten Interessengegenstand hin ausgerichtet ist (Schiefele 2009; Krapp und Prenzel 2011). Diesem Umstand wird in der Person-Gegenstands-Theorie des Interesses Rechnung getragen (Krapp 2002). Demnach entsteht Interesse als Wechselwirkung zwischen einer Person mit ihren individuellen Dispositionen und Fähigkeiten und einem Gegenstand. Als Gegenstand werden neben ganz konkreten, gegenständlichen Dingen auch beispielsweise politische oder gesellschaftliche Themen, (Schul-)Fächer oder auch abstrakte Vorstellungen bzw. kognitive Repräsentationen der Wirklichkeit verstanden (vgl. Krapp 2002; Krapp und Prenzel 2011).

Dieser Beitrag orientiert sich an aktuellen Arbeiten, welche zwischen individuellem und situationalem Interesse unterscheiden (Krapp 2002; Hidi und Renninger 2006; Schiefele 2009). Das individuelle oder dispositionale Interesse wird als relativ stabiles Persönlichkeitsmerkmal beschrieben, welches sich in der Bereitschaft widerspiegelt, sich mit einem Interessengegenstand (beispielsweise einem bestimmten Schulfach) auseinanderzusetzen (Krapp 2002; Schiefele 2009). Individuelles Interesse kann zu einer intrinsisch motivierten, wiederholten und zum Teil lebenslangen Auseinandersetzung mit dem Interessengegenstand führen (Krapp 1992, 2000). Das situationale Interesse wird hingegen als Zustand des an etwas Interessiertseins in einer bestimmten Situation beschrieben, welcher zu größerer Aufmerksamkeit, erhöhten kognitiven Funktionen und positiven Emotionen sowie zur Weckung von Neugier führt (Hidi und Renninger 2006; Schiefele 2009). Es kann durch ein bereits vorhandenes individuelles Interesse und/oder durch besondere Merkmale der Situation hervorgerufen werden (Krapp 2002). Dabei werden Situationen dann als besonders interessant empfunden, wenn sie von den Lernenden sowohl als neu als auch einigermaßen komplex sowie als verständlich eingeschätzt werden (Silvia 2008). Situationales Interesse ist jedoch nicht mit Interessantheit gleichzusetzen. Es entsteht vielmehr als Wechselwirkung von Dispositionen wie Vorwissen und individuellem Interesse des Lernenden sowie situativen Rahmenbedingungen bei der Auseinandersetzung mit einem Lerngegenstand (Krapp 2002; Hidi und Renninger 2006). Aktuelle Studienergebnisse stützen diese Sichtweise auf situationales Interesse als psychologischen Zustand (vgl. Knogler et al. 2015). Situationales Interesse kann von kurzer Dauer sein oder die Basis für ein langanhaltendes, individuelles Interesse darstellen (Krapp und Prenzel 2011).
Im Vier-Phasen-Modell der Interessenentwicklung beschreiben Hidi und Renninger (2006), wie sich aus einem typischerweise durch äußere Reize der Lernsituation geweckten situationalen Interesse (triggered situational interest) ein gefestigtes (maintained situationale interest) entwickelt (siehe auch Renninger und Hidi 2016). Daraus kann ein aufkeimendes (emerging individual interest) und schließlich ein ausgeprägtes individuelles Interesse resultieren (well-developed individual interest). Palmer et al. (2017) konnten in einer Längsschnittstudie die beiden von Hidi und Renninger (2006) beschriebenen Entwicklungsphasen des situationalen Interesses bestätigen und einen Zusammenhang zwischen einem immer wieder geweckten situationalen und einem sich entwickelnden individuellen Interesse an Naturwissenschaften nachweisen. Wenngleich sicherlich nicht aus jedem situationalen Interesse ein anhaltendes individuelles Interesse hervorgeht, so ist es doch eine notwendige Voraussetzung für die Interessensentwicklung: ,There are no data suggesting that individual interest emerges without first being experienced as a situational interest. " (Hidi und Renninger 2006, S. 117).

Interesse kann durch drei Komponenten charakterisiert werden (Krapp 2002; Renninger und Hidi 2011; Blankenburg und Scheersoi 2018). Die emotionale Komponente trägt dem Umstand Rechnung, dass eine Auseinandersetzung mit dem Interessengegenstand mit positiven Emotionen wie Neugier, Freude oder Flow-Erleben verbunden ist (vgl. Schiefele 2009). Des Weiteren wird der Interessengegenstand als persönlich bedeutsam betrachtet (wertbezogene Komponente), was unter anderem auch in der Bereitschaft resultiert, Aufwand wie Zeit oder Geld in die Interessenhandlung zu investieren (Blankenburg und Scheersoi 2018). Neben diesen eher affektiven Interessenskomponenten wird eine dritte beschrieben, welche den epistemischen Charakter von Interesse widerspiegelt, mehr über den Interessengegenstand lernen zu wollen (vgl. Krapp 2002; Hidi et al. 2004; Krüger und Vogt 2007).

Vielen Arbeiten zur Wirkung von Schülerlaborbesuchen auf das situationale Interesse liegt diese dreidimensionale Auffassung der Interessensstruktur zugrunde (vgl. Engeln 2004; Guderian et al. 2006; Pawek 2009; Streller 2015; Simon 2019). Verschiedene aktuelle Studien zu Kontextmerkmalen beziehen sich hingegen ausschließlich auf emotionale und wertbezogene Interessensaspekte (Fechner 2009; van Vorst 2013; Habig et al. 2018). Dies steht im Einklang mit neueren Arbeiten zum Interessenskonstrukt, welche in erster Linie emotionale und/oder wertbezogene Valenzen beschreiben (Silvia 2008; Schiefele 2009; Alexander und Grossnickle 2016). Eine wichtige, auch unterrichtsrelevante Rolle bei der Entwicklung von individuellem Interesse spielt aber gerade der Wunsch mehr über den Interessengegenstand lernen zu wollen (vgl. Krapp 2002; Hidi et al. 2004; Renninger und Su 2012). Diese epistemi- 
sche Komponente des situationalen Interesses ist darüber hinaus auch aus praktischer Sicht von Bedeutung. So kann ein ausgeprägtes situationales epistemisches Interesse auch Aufschluss darüber geben, inwiefern Lerninhalte die Schüler*innen ansprechen oder nicht (vgl. Priemer et al. 2018). Daher orientiert sich dieser Beitrag an einer dreidimensionalen Struktur des situationalen Interesses. Ob sich aus einem situationalen Interesse ein längerfristig anhaltendes individuelles Interesse entwickelt, hängt auch davon ab, inwiefern der Interessengegenstand als etwas persönlich Sinnvolles und Bedeutsames wahrgenommen wird (vgl. Mitchell 1993; Prenzel und Drechsel 1996; Schiefele 2009; Edelmann und Wittmann 2012). Ein Maß für diese empfundene Bedeutsamkeit ist die wahrgenommene inhaltliche Relevanz.

\section{Inhaltliche Relevanz}

Der Begriff Relevanz wird im Zusammenhang mit naturwissenschaftlichem Unterricht nicht einheitlich verwendet. Laut einer internationalen Literaturanalyse von Stuckey et al. (2013) betrachten manche Autoren Relevanz als Synonym für Interesse, andere als $\mathrm{Ma}$ dafür, wie sinnvoll es Lernenden erscheint, lebensweltbezogene Kontexte zu verstehen. Wieder andere verstehen Relevanz als Synonym für die Wichtigkeit oder Nützlichkeit der Inhalte für die Lernenden. Der Begriff kann auch im Sinne realer Auswirkungen für den Einzelnen oder die Gesellschaft verstanden werden - teilweise finden sich aber auch Kombinationen aus den genannten Vorstellungen (Stuckey et al. 2013). In diesem Beitrag wird inhaltliche Relevanz im Sinne von Prenzel und Drechsel (1996) bzw. Seidel et al. (2005) bezogen auf Alltagsbedeutung, praktische Nutzbarkeit und Realitätsnähe verstanden.

Lewalter und Geyer (2009) konnten im Einklang mit der Person-Gegenstands-Theorie des Interesses empirisch belegen, dass die wahrgenommene inhaltliche Relevanz im oben genannten Sinne stark mit dem situationalen Interesse von Schüler*innen beim Besuch von naturwissenschaftlich-technischen Museen korreliert. Demnach stehen insbesondere interessestabilisierende Aspekte (Hold-Faktoren, vgl. Mitchell 1993) in engem Zusammenhang mit der wahrgenommenen inhaltlichen Relevanz. Lewalter und Geyer (2009) weisen aber auch darauf hin, dass zur Klärung der Frage, welche konkreten Gestaltungskriterien zur Relevanzwahrnehmung beitragen, weitere Analysen erforderlich sind. Eine Studie von Watzka und Girwidz (2015) belegt, dass die Wahrnehmung von inhaltlicher Relevanz durch Lernen in einem authentischen Kontext stärker angesprochen wird als durch einen weniger stark authentischen Kontext. Die wahrgenommene inhaltliche Relevanz korreliert dabei stark bis sehr stark mit der wahrgenommenen Authentizität. Authentische Kontexte begünstigen demnach die Wahrnehmung von inhaltlicher Relevanz und damit einhergehend auch anderer, lernförderlicher Faktoren.

\section{Authentizität}

Auch der Begriff Authentizität bedarf einer genaueren Klärung, da er oft in unterschiedlichen Bedeutungen verwendet wird (Anker-Hansen und Andreé 2019). Van Vorst et al. (2015, S. 32) betrachten Authentizität als ,ein Merkmal, das sich der Interaktion zwischen Schülerin bzw. Schüler und Kontext zuordnen lässt" und insbesondere dessen Glaubwürdigkeit vermittelt. In diesem Sinne kann Authentizität als eine Art subjektive Einschätzung der wahrgenommenen Echtheit verstanden werden. Das Authentizitätsmodell von Betz et al. (2016) sowie Betz (2018) differenziert diese Betrachtungsweise weiter aus. Demnach treffen Lernende mit individuellen Personenmerkmalen wie Vorwissen, epistemologischen Überzeugungen und individuellen Interessen auf eine Lernsituation mit eher objektiven Merkmalen des Lernsettings (Lernort und -material, Vermittler, Inhalt etc.). Bei der Auseinandersetzung mit dem Lerngegenstand erlebt jeder Lernende ein individuelles Authentizitätsempfinden, das affektive, kognitive und verhaltensbezogene Effekte verursachen kann. Dieser Beitrag folgt im Wesentlichen dieser Vorstellung von Authentizität als Wechselwirkung aus objektiven Rahmenbedingungen der Lernumgebung und der individuellen Wahrnehmung der Situation.

Die mancherorts etwas unklare Verwendung des Begriffs Authentizität ist besonders problematisch, da dieser in der Alltagssprache positiv konnotiert ist und somit eine authentische Lernumgebung etwas unreflektiert per se als besser angenommen werden könnte, als eine weniger authentische (vgl. auch Höttecke und Rieß 2015). Davon kann so pauschal sicherlich nicht ausgegangen werden, aber es gibt vielfache empirische Evidenz dafür, dass authentische Kontexte bzw. Lernumgebungen unter bestimmten Umständen lern- und interesseförderlich wirken können (u.a. Bennett et al. 2007; Lewalter und Geyer 2009; Kuhn 2010; Watzka und Girwidz 2015; Habig et al. 2018; Betz 2018). Eine mögliche Erklärung für die interesseförderliche Wirkung einer authentischen Lernumgebung kann im Einklang mit der Person-Gegenstands-Theorie gegeben werden: Ein authentischer Kontext bzw. eine authentische Umgebung als Interessengegenstand eignen sich unter bestimmten Umständen dafür, die Wahrnehmung inhaltlicher Relevanz zu steigern (vgl. Lewalter und Geyer 2009; Watzka und Girwidz 2015). Die wahrgenommene inhaltliche Relevanz kann aus theoretischer Sicht als interesseförderlicher Faktor identifiziert werden (Schiefele 2009; Parchmann und Kuhn 2018). Ein enger positiver Zusammenhang der inhaltlichen Relevanz insbesondere mit interessestabilisierenden Aspekten des situationalen Interesses ist empirisch belegt (Lewalter und Geyer 2009). 
In der vorliegenden Arbeit wird überprüft, inwiefern sich unterschiedlich authentische Lernsettings (Lernort und -material) auf das situationale Interesse sowie die Wahrnehmung von inhaltlicher Relevanz und von Authentizität auswirken. Als Lernort wurde hierzu ein etabliertes, wissenschaftsnahes, klassisches Schülerlabor am Deutschen Zentrum für Luft- und Raumfahrt gewählt (vgl. Euler und Schüttler 2020).

\section{Schülerlabore als interessefördernde, authentische Lernorte}

In den vergangenen gut 20 Jahren haben sich Schülerlabore als außerschulische Lernorte fest in der deutschen Bildungslandschaft etabliert (Euler und Schüttler 2020; Pawek 2019). Als primäre Ziele werden insbesondere die Förderung von Interesse an und Verständnis für Naturwissenschaften sowie Berufsorientierung und Nachwuchsförderung genannt (Haupt et al. 2013). Als Einflussgrößen für die Wirkung von Schülerlaboren wird der Authentizität eine herausragende Bedeutung zugesprochen. Scharfenberg und Bogner (2015, S. 24) nennen sie in diesem Zusammenhang „entscheidend“, Euler et al. (2015, S. 762) sprechen gar vom „Trumpf der Schülerlabore“. Dem stellen Priemer und Lewalter (2009) entgegen, dass sich die Einrichtungen in der wirklichen Forschungsnähe, also der Authentizität im Sinne der Nähe zur echten naturwissenschaftlichen Forschung, zum Teil stark unterscheiden. Demnach stellen also nicht alle Schülerlabore gleichermaßen authentische Lernsettings bereit. Klassische, wissenschaftsnahe Schülerlabore an Forschungseinrichtungen ${ }^{1}$ nutzen die räumliche, technische und thematische Nähe zur echten Forschung, um möglichst authentische Einblicke zu vermitteln (Euler und Schüttler 2020).

Die Wirkung von Schülerlaborbesuchen auf das Interesse ihrer Besucher wird in Überblicksdarstellungen überwiegend positiv bewertet (Priemer und Lewalter 2009; Euler et al. 2015; Scharfenberg und Bogner 2015; Priemer et al. 2018; Pawek 2019). Das situationale Interesse der Teilnehmer*innen an den Veranstaltungen wird auf deskriptiver Ebene für die emotionale und die wertbezogene Interessenskomponente als hoch bis sehr hoch beschrieben. Auch auf andere affektive Lernmerkmale wirken sich die Laborbesuche in den meisten untersuchten Fällen positiv aus. Die epistemische Komponente des situationalen Interesses wird in der Schülerlaborforschung hingegen oft als weniger hoch berichtet (vgl. Priemer et al. 2018). Dies ist bedenklich,

\footnotetext{
${ }^{1}$ Der Begriff „klassisches Schülerlabor“ wird nicht einheitlich verwendet. In diesem Beitrag folgen wir der Auffassung von Pawek (2019). Anstelle der sehr umfassenden Kategorisierung von Haupt et al. (2013), bezeichnet er Schülerlabore an Hochschulen und Forschungseinrichtungen mit starkem Bezug zur aktuellen Forschung in Abgrenzung zu Lehr-Lern-Laboren als ,klassisch“.
}

da epistemische Interessensaspekte als besonders wichtig für die Entwicklung von individuellem Interesse angesehen werden können (vgl. Renninger und Su 2012). Insbesondere stark unterrichtsnah ausgelegte Labore bleiben in diesem Aspekt teilweise hinter den Erwartungen zurück und erzielen deutlich niedrigere Werte als eher offen und wissenschaftsnah gestaltete Labore (Euler und Weßnigk 2011).

Auf der Seite der Lernenden hat das Sachinteresse an Naturwissenschaften, einen signifikanten Einfluss auf das situationale epistemische Interesse (Pawek 2009). Geschlechterunterschiede wurden in diesem Zusammenhang hingegen mehrfach als wenig bedeutsam berichtet: Priemer et al. (2018) fanden in einer groß angelegten Studie $(N=11.032)$ keine Unterschiede zwischen Jungen und Mädchen im situationalen epistemischen Interesse beim Schülerlaborbesuch. Auch andere Studien in Schülerlaboren kommen bezüglich Gendereffekten für das situationale Interesse zu ähnlichen Ergebnissen (vgl. Pawek 2019). Auf der Seite der Labore konnte ein moderater, signifikanter Zusammenhang zwischen der wahrgenommenen Authentizität der Forschungsumgebung und einem vergleichsweise hohen situationalen epistemischen Interesse nachgewiesen werden (Pawek 2009). Allerdings bleibt offen, worauf die wahrgenommene Authentizität zurückzuführen ist - inwiefern also der besonders authentische Lernort oder die im Schülerlabor eingesetzten Materialien diese vermitteln oder ob andere Faktoren von Bedeutung sind. Dies erschwert eine Übertragbarkeit in andere Lernsettings. Ähnliches gilt für eine Studie von Glowinski (2007).

In Bezug auf den Lernzuwachs bei einem lehrplanbezogenen, schulnahen Thema konnten in einer relativ großen Vergleichsstudie $(N=1773)$ keine signifikanten Unterschiede nach einem Unterricht am authentischen Lernort Schülerlabor gegenüber einem zu denselben Inhalten an Schulen gehaltenen Unterricht festgestellt werden (Itzek-Greulich et al. 2015). Die wahrgenommene Authentizität der Lernumgebung als Kombination aus authentischem Lernort und vermittelnder Person (Wissenschaftler als zusätzlicher Betreuer) erwies sich jedoch bei Schülerlaborbesuchen im Vergleich zur selben Aktivität in der Schule ohne zusätzlichen Wissenschaftler als stärker ausgeprägt und förderlich für das situationale Interesse (Betz 2018). Die Übertragbarkeit der Ergebnisse auf andere wissenschaftliche Disziplinen als die untersuchte (Linguistik) wird von der Autorin allerdings als problematisch bezeichnet. Weitere empirische Vergleichsstudien aus diesem Forschungsverbund, die explizit die Bedeutung der Authentizität beim Schülerlaborbesuch im Fokus hatten, sind aufgrund der spezifischen geschichts- bzw. erziehungswissenschaftlichen Fragestellungen zwar nicht direkt auf den naturwissenschaftlichen Unterricht übertragbar, geben aber weitere Hinweise auf die Wirkung der Authentizität beim Schülerlaborbesuch. So führte der Einsatz authentischer Materialien im 
Vergleich zu nicht-authentischen zu einer signifikanten $\mathrm{Zu}$ nahme einer wissenschaftlich adäquaten epistemologischen Überzeugungshaltung (Mierwald et al. 2018). Eine authentischere Lernaktivität, die sich stärker an typischen wissenschaftlichen Arbeitsweisen orientierte, zeigte gegenüber einer weniger authentischen hingegen weder Effekte auf die wahrgenommene Authentizität noch auf das situationale Interesse (Nachtigall et al. 2018).

Für Schülerlabore mit naturwissenschaftlichen Themen wird der Einsatz echter Laborgeräte als wichtiger Erfolgsfaktor angesehen (Hausamann 2012). Allerdings fehlen bislang entsprechende Vergleichsstudien. In der Schule konnte beobachtet werden, dass eigenständiges Experimentieren interesseförderlich wirken kann (Holstermann et al. 2010). Jedoch war diese Wirkung nur für einige der untersuchten Aktivitäten aus dem Biologieunterricht feststellbar. Bekannt ist auch, dass Schüler*innen den für sie neuen Geräten aus der schulischen Sammlung gegenüber Alltagsmaterial den Vorzug geben (Behrendt 1990). Allerdings sind die in Schülerlaboren eingesetzten Instrumente aus der echten Forschung nicht vergleichbar mit den für Bildungszwecke optimierten Geräten aus Schulsammlungen. Sie sind zwar höchst authentisch für die jeweilige Forschung aber auch relativ komplex und können oft nur durch entsprechend geschulte Personen bedient werden. Es ist zudem unklar, ob derartige Instrumente von den Besuchern der Labore überhaupt als authentisch für die dort stattfindende Forschung wahrgenommen werden.

Die derzeit vorliegenden empirischen Studien zur Bedeutung der wahrgenommenen Authentizität für das situationale Interesse geben Grund zur Annahme, dass sich eine möglichst authentische Lernumgebung positiv auswirken kann (Itzek-Greulich und Vollmer 2018). Für naturwissenschaftlich orientierte Lernsettings fehlt jedoch bislang ein entsprechender, direkter Vergleich unterschiedlich authentischer Lernorte (Schule vs. Schülerlabor) und Laborgeräte (High-End-Laborgeräte vs. Low-Cost-Geräte) unter sonst möglichst gleichen Bedingungen.

\section{Forschungsfrage und Hypothese}

Durch eine Fokussierung auf die Authentizität des Lernortes und der Laborgeräte bei sonst möglichst konstanten Bedingungen sollte diese Studie personenunabhängige, auch auf den Schulunterricht übertragbare Ergebnisse liefern. Konkret wurde folgende Frage untersucht:
Inwiefern unterscheiden sich das situationale Interesse und die Wahrnehmung von inhaltlicher Relevanz und Authentizität in Abhängigkeit vom Einsatz unterschiedlich authentischer Laborgeräte an unterschiedlich authentischen Lernorten unter Kontrolle des Sachinteresses an Naturwissenschaften und des Geschlechts?

Aufbauend auf den eingangs beschriebenen theoretischen Überlegungen und empirischen Befunden wird folgende Hypothese formuliert:

Die Authentizität des Lernsettings (Ort und Laborgeräte) wird als prädiktiv für das situationale Interesse, insbesondere für die epistemische Komponente, angenommen. Zudem wird erwartet, dass im authentischeren Lernsetting (Schülerlabor mit High-End-Laborgeräten) eine größere Relevanz- und Authentizitätswahrnehmung zu beobachten ist als in den weniger authentischen Lernsettings (Schülerlabor mit Low-Cost-Geräten, Schule mit High-End-Laborgeräten, Schule mit Low-Cost-Geräten).

\section{Methode}

\section{Studiendesign und Variablen}

Bei dieser quasi-experimentellen Studie wurde ein $2 \times 2$ faktorielles Design gewählt. Als unabhängige Variablen (UV) gingen ein: Authentizität des Lernorts (Schülerlabor am Deutschen Zentrum für Luft- und Raumfahrt (DLR) vs. Schule) und der Laborgeräte (High-End vs. Low-Cost). Bei der Studie wurden in einem Prätest als Kontrollvariablen u.a. das Geschlecht sowie das Sachinteresse an Naturwissenschaften erhoben. Als abhängige Variablen (AV) wurden im Posttest u. a. die epistemische, emotionale und wertbezogene Komponente des situationalen Interesses, die wahrgenommene inhaltliche Relevanz sowie die wahrgenommene Authentizität der Laborgeräte gemessen. Die Studie fand im Schuljahr 2017/18 statt.

\section{Stichprobe}

An der Studie beteiligten sich insgesamt 166 Zehntklässler*innen aus vier Gymnasien im Großraum München. Zur Zeit der Befragung waren die Studienteilnehmer zwischen 15 und 17 Jahren alt ( $M=15,8$ Jahre; $S D=0,6$ Jahre). Insgesamt liegen von $N=148$ Probanden vollständig ausgefüllte Fragebögen beider Befragungszeitpunkte vor. Nur diese wurden in der Auswertung berücksichtigt. Die Schü-

Tab. 1 Probandenverteilung der Stichprobe

\begin{tabular}{|c|c|c|c|c|c|c|c|c|}
\hline \multirow{3}{*}{$\begin{array}{l}\text { Authentizität d. Lernorts } \\
\text { Authentizität d. Laborgeräte } \\
\text { Geschlecht }\end{array}$} & \multicolumn{4}{|c|}{ Schülerlabor } & \multicolumn{4}{|c|}{ Schule } \\
\hline & \multicolumn{2}{|c|}{ High-End } & \multicolumn{2}{|c|}{ Low-Cost } & \multicolumn{2}{|c|}{ High-End } & \multicolumn{2}{|c|}{ Low-Cost } \\
\hline & $\bar{M}$ & $\mathrm{~W}$ & $\bar{M}$ & $\mathrm{~W}$ & $\mathrm{M}$ & $\mathrm{W}$ & $\mathrm{M}$ & $\mathrm{W}$ \\
\hline $\bar{N}$ & 26 & 10 & 15 & 22 & 26 & 14 & 21 & 14 \\
\hline
\end{tabular}


Tab. 2 Überblick der eingesetzten Skalen mit Beispielitems

\begin{tabular}{|c|c|c|c|c|c|}
\hline Testzeitpunkt & \multicolumn{2}{|l|}{ Skala } & Itemanzahl & Beispielitem & Cronbach's $\alpha$ \\
\hline Prätest (T1) & \multicolumn{2}{|c|}{$\begin{array}{l}\text { Sachinteresse an } \\
\text { Naturwissenschaften }\end{array}$} & 6 & Naturwissenschaften machen mir Spaß ${ }^{a}$ & 0,85 \\
\hline \multirow[t]{5}{*}{$\begin{array}{l}\text { Posttest } \\
\text { (T2) }\end{array}$} & \multirow[t]{3}{*}{$\begin{array}{l}\text { Situationales } \\
\text { Interesse }\end{array}$} & Epistemisch & 5 & $\begin{array}{l}\text { Ich würde gerne mehr über die Experimente lernen, die } \\
\text { wir im Schülerlabor durchgeführt haben }\end{array}$ & 0,76 \\
\hline & & Emotional & 5 & Die Experimente waren für mich interessant & 0,74 \\
\hline & & Wertbezogen & 4 & Das eigenständige Experimentieren war mir wichtig & 0,60 \\
\hline & \multicolumn{2}{|c|}{ Inhaltliche Relevanz } & 3 & $\begin{array}{l}\text { Im „Schülerlabor“ lernten wir, wo die Inhalte praktisch } \\
\text { angewendet werden }\end{array}$ & 0,70 \\
\hline & \multicolumn{2}{|c|}{$\begin{array}{l}\text { Wahrgenommene Authentizität der } \\
\text { Laborgeräte }\end{array}$} & 4 & $\begin{array}{l}\text { Die Experimentiermaterialien, die ich heute verwenden } \\
\text { konnte, werden auch von Fachleuten in der Forschung } \\
\text { eingesetzt }\end{array}$ & 0,85 \\
\hline
\end{tabular}

Die Werte für Cronbach's $\alpha$ können fast durchweg als akzeptabel bis gut bezeichnet werden

aDie Formulierung bei Pawek (2009, S. 195) lautet: „Naturwissenschaften bringen mir Spaß.“ In einer Vorstudie zur Evaluierung der eingesetzten Technik (vgl. Schüttler et al. 2019) führte diese Formulierung jedoch zu Verständnisproblemen der Schüler*innen, weshalb das Wort „bringen“ durch „machen“ ersetzt wurde

ler*innen nahmen jeweils im Klassenverbund an der Studie teil. Sie wurden auf die vier Versuchsgruppen so aufgeteilt, dass diese in etwa gleich groß waren (vgl. Tab. 1). Die Verteilung der Probanden war bei der hier durchgeführten quasiexperimentellen Feldstudie stark durch die Klassenzusammensetzung beeinflusst. Ein Chi-Quadrat-Anpassungstest zeigt, dass die Geschlechter in den Gruppen nicht gleichverteilt waren $\left(\chi^{2}[3,148]=8,44 ; p=0,038\right)$. Besonders auffällig ist die asymmetrische Geschlechterverteilung der Gruppen im Schülerlabor. Um mögliche Unterschiede in der Wahrnehmung der Mädchen und Jungen zu kontrollieren, wurde das Geschlecht daher in der Auswertung der Daten berücksichtigt. Eine Beschreibung der entsprechenden Ergebnisse ist im Anhang zu finden.

Um Klasseneffekte zu reduzieren, wurden aus den vier Schulen jeweils zwei Parallelklassen gleicher Profilausrichtung (sprachlich oder naturwissenschaftlich) - insgesamt also acht Klassen - gewählt, die von den unterrichtenden Lehrkräften als vergleichbar in ihrem Interesse an naturwissenschaftlichen Fächern beschrieben wurden. Die Ergebnisse anderer Studien (Pawek 2009) und Erfahrungen mit einer Vorstudie (Bohnhardt und Schüttler 2020) legten nahe, zusätzlich zu dieser Einschätzung der Lehrkräfte im Prä-Test Kontrollvariablen zu erheben, um die Homogenität der Stichprobe zu kontrollieren. Hierzu wurde, auf Basis der Ergebnisse ähnlicher Studien (insbes. Pawek 2009; sowie Lewalter und Geyer 2009), zusätzlich zum Geschlecht auch das Sachinteresse an Naturwissenschaften (vgl. Erhebungsinstrumente) als Kontrollvariable ausgewählt.

\section{Erhebungsinstrumente}

Die Entwicklung der Fragebögen erfolgte unter Verwendung bereits bestehender Instrumente, ergänzt durch selbst formulierte Items. Die eingesetzten Rating-Skalen reichen von (1) „trifft nicht zu“ bis (5) „trifft völlig zu“ (vgl.
Tab. 2). Die Skala zum Sachinteresse an Naturwissenschaften stammt von Köller et al. (2000) und wurde in der von Pawek (2009) auf Basis von Engeln (2004) leicht veränderten Form verwendet ${ }^{2}$. Die interne Konsistenz ist mit Cronbach's $\alpha=0,85$ als gut zu bezeichnen (vgl. Kuckartz et al. 2013). Die Skala zum situationalen Interesse wurde im Wortlaut von Pawek (2009) übernommen. Die zugrundeliegende Skala von Engeln (2004) wurde bereits vielfach zur Messung von situationalem Interesse eingesetzt (u.a. Guderian et al. 2006; Fechner 2009; Pawek 2009; van Vorst 2013; Streller 2015; Habig et al. 2018; Priemer et al. 2018). Die interne Konsistenz der Skala ist mit Cronbach's $\alpha$ Werten größtenteils über 0,7 als akzeptabel zu bezeichnen. Lediglich bei der wertbezogenen Komponente des situationalen Interesses wurden mit $\alpha=0,60$ ähnlich niedrige Werte gemessen, wie bei anderen Studien (Pawek 2009). Zugunsten der Vergleichbarkeit unterblieben hier Anpassungen. Um die Trennung der drei Interessensdimensionen statistisch abzusichern wurde die Skala mit einer konfirmatorischen Faktorenanalyse überprüft. Die Ergebnisse weisen auf eine akzeptable bis gute Modellpassung hin $\left(\chi^{2} / d f=1,6 ; \quad C F I=0,912 ; R M S E A=0,063 ; S R M R=0,063\right.$; vgl. Weiber und Mühlhaus 2014; und Anhang). Die wahrgenommene inhaltliche Relevanz wurde mit einer von Seidel et al. (2005) entwickelten Skala (vgl. Skalenhandbuch der PISA-Studie 2006 [Frey et al. 2009]) gemessen und weist eine noch akzeptable interne Konsistenz auf (Cronbach's $\alpha=0,70$ ). Die Skala zur wahrgenommenen Authentizität der Laborgeräte wurde neu entwickelt und ist intern konsistent (Cronbach's $\alpha=0,85$ ).

\footnotetext{
2 Die Skala besteht bei Pawek (2009) aus sieben Items. Auf ein sehr negativ formuliertes (,In meiner Freizeit habe ich besseres zu tun, als über naturwissenschaftliche Phänomene nachzudenken. " Pawek 2009, S. 195) wurde hier verzichtet.
} 
Abb. 1 Die Probanden erhielten an unterschiedlich authentischen Lernorten (Schülerlabor des DLR bzw. Schule) inhaltsgleiche Workshops, die sich im Wesentlichen nur durch die Authentizität der Laborgeräte (High-End bzw. Low-Cost) unterschieden. Die Betreuung war an beiden Orten identisch. Unmittelbar vor (T1) und nach (T2) der Intervention wurden sie mit Fragebögen befragt

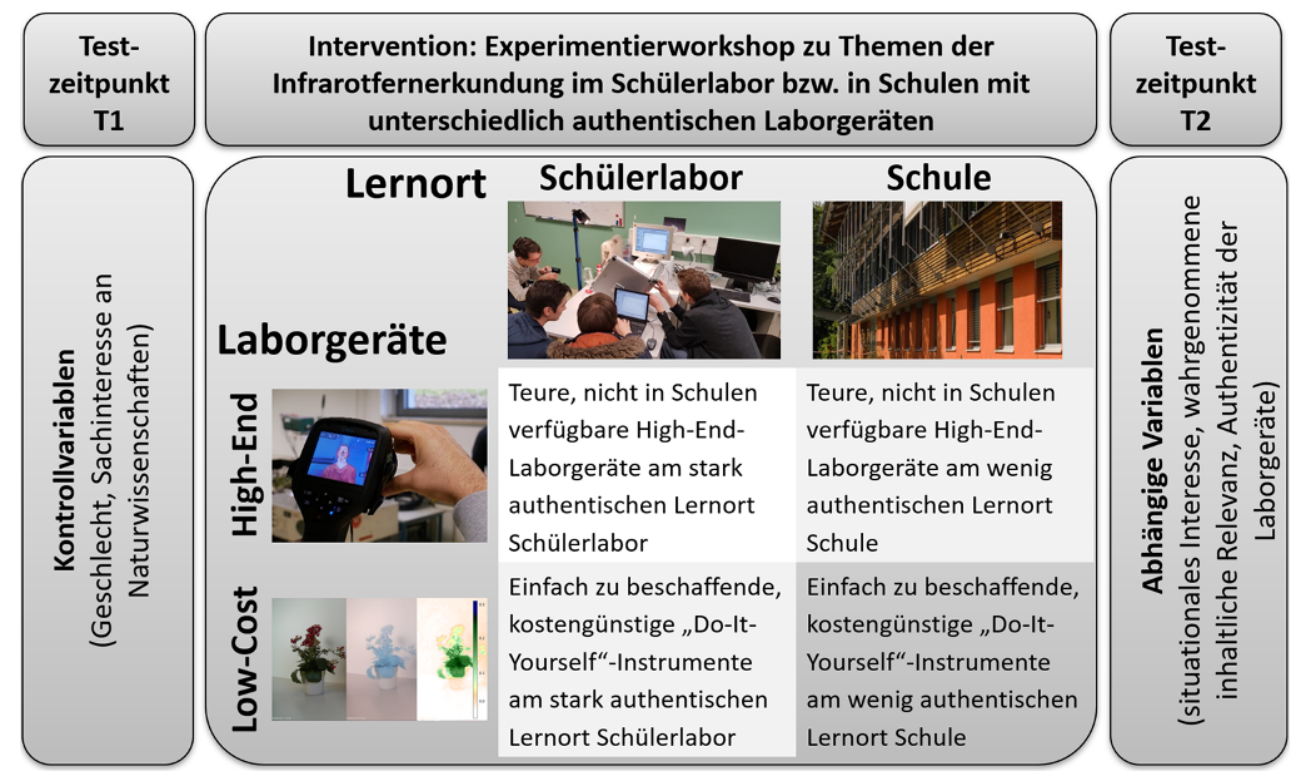

\section{Ablauf der Studie}

Der Untersuchungszeitraum wurde auf zwei aufeinanderfolgende Wochen terminiert, um einen möglichst vergleichbaren Entwicklungsstand der Proband*innen sicher zu stellen. Alle Schüler*innen wurden zu Beginn des Tages, direkt vor dem Einführungsvortrag (TI) und am Ende, direkt nach der zweiten Experimentierphase (T2) mit einem Fragebogen befragt. Die Durchführung der Erhebung wurde in allen Fällen standardisiert durch den Autor vorgenommen (vgl. Abb. 1). In Absprache mit den Lehrkräften erhielten die Studienteilnehmer*innen keine Vorinformationen zum durchgeführten Programm, um nicht durch unterschiedliche Erwartungen die Ergebnisse der ersten Befragung zu beeinflussen.

Der Workshop zu Themen der Infrarotfernerkundung begann mit einem 30-minütigen Einführungsvortrag dem zwei 90-minütige Experimentierphasen in Kleingruppen mit je 4-6 Schüler*innen folgten. Er basiert auf den zwei etablierten Experimenten „Infrarotmesstechnik“ und „Optische Umweltfernerkundung“ des DLR_School_Lab Oberpfaffenhofen (vgl. Locherer et al. 2012). Sie behandeln strahlungsphysikalische Themen, die eingebettet sind in die Kontexte Fernerkundung von Hochtemperaturereignissen wie Waldbränden und Fernerkundung von Vegetation mit spektroskopischen Verfahren. Neben den Anwendungsfeldern und wissenschaftlichen Hintergründen erwerben die Teilnehmer*innen auch die physikalischen Grundlagen (vgl. www.dlr.de/schoollab/oberpfaffenhofen).

Der Workshop wurde an unterschiedlich authentischen Orten (Schülerlabor bzw. Schule) jeweils mit unterschiedlich authentischen Laborgeräten (High-End bzw. Low-Cost) durchgeführt (vgl. Abb. 1). Bei der Konzeption der Sta- tionen wurde besonders darauf geachtet, dass sich diese möglichst nur in den eingesetzten Laborgeräten, nicht aber in den behandelten Inhalten und Herangehensweisen unterschieden. Kleinere Anpassungen im Ablauf waren aufgrund der unterschiedlichen Funktionalitäten der Instrumente erforderlich. Betreut wurden die Proband*innen immer von denselben drei erfahrenen Mitarbeiterinnen und Mitarbeitern des DLR_School_Lab und vom Autor selbst. Um damit möglicherweise einhergehende Lehrer- bzw. Versuchsleitereffekte (vgl. Brosius et al. 2012) möglichst auszuschließen, wurde sichergestellt, dass die Rolle der Betreuer von untergeordneter Bedeutung war. Dazu wurden vorab präzise Absprachen getroffen und ein Skript verwendet, das den Unterricht standardisierte (vgl. Döring und Bortz 2016). Die Vorgehensweise wurde in einer Vorstudie erarbeitet (Bohnhardt und Schüttler 2020). Zudem lag der Schwerpunkt auf einem schülerorientierten Lernen in Kleingruppen, das so angeleitet wurde, dass die SuS größtenteils selbstständig experimentieren konnten.

\section{Authentizität des Ortes}

Am DLR in Oberpfaffenhofen befasst sich eine Vielzahl von Wissenschaftler*innen mit der Satellitenfernerkundung (vgl. https://www.dlr.de/eoc). Das Schülerlabor befindet sich in ehemaligen Satellitenkontrollräumen und Labors, sodass man hier von einem besonders authentischen Lernort für das behandelte Thema sprechen kann. Kontrastierend wurde der Workshop an Schulen als wenig authentischem Ort durchgeführt. Die Ergebnisse von Betz (2018) und die offensichtlich besondere Umgebung des Schülerlabors legen nahe, dass dieser Ort als authentisch wahrgenom- 
men wird, weshalb auf die Kontrolle dieser Wahrnehmung verzichtet wurde.

\section{Authentizität der Laborgeräte}

Im DLR_School_Lab sollen neben dem Ort spezielle Messinstrumente und Laborgeräte, wie sie auch in den entsprechenden Instituten des DLR zum Einsatz kommen, bei den Lernenden eine besonders starke Authentizitätswahrnehmung bewirken (Euler und Schüttler 2020). Bei den authentischen Laborgeräten (High-End) des hier beschriebenen Workshops handelt es sich insbesondere um hochauflösende Thermalinfrarotkameras und ein sehr präzises, wissenschaftliches Feldspektrometer. Als für die Forschung wenig authentische Alternative wurden kostengünstige, einfach zu beschaffende und zum Teil selbstgebaute Instrumente bereitgestellt (Low-Cost). Die Maßgabe war, dass damit dieselben Inhalte und Themen wie mit den echten Laborgeräten behandelt werden konnten, und dass sie für Schulen verfügbar, dabei aber ausreichend präzise, ansprechend und gut $\mathrm{zu}$ bedienen waren. Die zugrunde liegende Technik wurde daher ausgiebig auch mit Schüler*innen erprobt und die Messdaten validiert (Schüttler et al. 2017, 2018). Dabei erwiesen sich die Geräte als für Bildungszwecke ausreichend genau und für Schülerprojekte geeignet (Schüttler et al. 2019). Mit ihnen ist es möglich, aktuelle Methoden der Satellitenfernerkundung für Schulen experimentell zugänglich zu machen (Schüttler und Girwidz 2019; Schüttler und Zepp 2019).

\section{Eingesetzte statistische Verfahren}

Die Auswertung aller Daten erfolgte mit der Statistiksoftware RStudio (2020) unter Verwendung der Pakete psych, readxl, effsize, xtable, dplyr, lawstat, lavaan, ggpubr, jmv und Hmisc. Aus den Items der Skalen wurden durch Mittelwertbildung Scores für die entsprechenden Variablen berechnet, deren Mittelwerte und Standardabweichungen in Tab. 3 wiedergegeben sind. Die Vorrausetzungen für die parametrischen Tests wurden überprüft. Dabei wurden keine multivariaten Ausreißer gefunden, gemessen durch die Mahalanobis-Distanz ( $p>0,001$ der Chi-Quadrat-Verteilung). Multinormalverteilung wurde durch Mardia-Kurtosis-Test abgesichert ( $p=0,607)$. Multikollinearität ist bei den in Tab. 4 angegebenen Korrelationskoeffizienten nicht zu erwarten ( $r<0,9$; vgl. Tabachnick und Fidell 2012). Homogenität der Kovarianzenmatrizen war gemäß Box-MTest gegeben ( $p>0,01$; vgl. Mertler und Reinhart 2017).

Um Zusammenhänge zwischen den AV untereinander und mit der Kovariaten Sachinteresse an Naturwissenschaften zu erfassen, wurden die Korrelationskoeffizienten (Pearson's r) berechnet und auf statistische Signifikanz geprüft. Anschließend wurde eine Multivariate Kovarianzanalyse (MANCOVA) durchgeführt, um die Haupteffekte der UV auf die AV unter Berücksichtigung der Kovariate (Sachinteresse an Naturwissenschaften) zu untersuchen. Die Ergebnisse der MANCOVA weisen signifikante Unterschiede auf, sodass auch tiefergehende Analysen der zugehörigen ANCOVAs zulässig sind (Tabachnick und Fi-

Tab. 3 Kennwerte für das im Prätest (T1) erhobene Sachinteresse an Naturwissenschaften sowie die im Posttest (T2) gemessenen Variablen (Komponenten des situationalen Interesses, wahrgenommene inhaltliche Relevanz und wahrgenommene Authentizität der Laborgeräte)

\begin{tabular}{|c|c|c|c|c|c|c|c|c|c|c|}
\hline \multirow[t]{3}{*}{ Zeitpunkt } & \multirow{2}{*}{\multicolumn{2}{|c|}{$\begin{array}{l}\text { Lernort (UV) } \\
\text { Laborgeräte (UV) }\end{array}$}} & \multicolumn{4}{|c|}{ Schülerlabor } & \multicolumn{4}{|c|}{ Schule } \\
\hline & & & \multicolumn{2}{|c|}{ High-End } & \multicolumn{2}{|c|}{ Low-Cost } & \multicolumn{2}{|c|}{ High-End } & \multicolumn{2}{|c|}{ Low-Cost } \\
\hline & & & $\overline{\mathrm{M}}$ & $\mathrm{SD}$ & $\bar{M}$ & SD & $\overline{\mathrm{M}}$ & SD & $\overline{\mathrm{M}}$ & SD \\
\hline $\mathrm{T} 1$ & \multicolumn{2}{|c|}{ Sachinteresse an Naturwissenschaften } & 3,50 & 0,89 & 3,34 & 0,74 & 3,19 & 0,78 & 2,96 & 0,73 \\
\hline \multirow[t]{5}{*}{$\mathrm{T} 2$} & \multirow{3}{*}{$\begin{array}{l}\text { Situationales } \\
\text { Interesse }\end{array}$} & Epistemisch & 3,49 & 0,77 & 3,03 & 0,59 & 3,04 & 0,61 & 3,01 & 0,78 \\
\hline & & Emotional & 4,24 & 0,57 & 4,02 & 0,56 & 4,18 & 0,52 & 4,07 & 0,56 \\
\hline & & Wertbezogen & 3,92 & 0,60 & 3,99 & 0,49 & 3,93 & 0,56 & 3,89 & 0,70 \\
\hline & \multicolumn{2}{|c|}{ Wahrgenommene inhaltliche Relevanz } & 3,80 & 0,73 & 3,63 & 0,60 & 3,35 & 0,73 & 3,33 & 0,75 \\
\hline & \multicolumn{2}{|c|}{ Wahrgenommene Authentizität der Laborgeräte } & 4,36 & 0,56 & 3,32 & 0,85 & 4,31 & 0,73 & 3,05 & 0,82 \\
\hline
\end{tabular}

Tab. 4 Bivariate Korrelationen (Pearson's $r$ ) der abhängigen Variablen und der Kovariate Sachinteresse an Naturwissenschaften

\begin{tabular}{|c|c|c|c|c|c|c|}
\hline- & (1) & (2) & (3) & (4) & (5) & (6) \\
\hline Sachinteresse Naturwissenschaften (1) & 1,00 & $0,49 * * *$ & $0,39 * * *$ & 0,13 & $0,50 * * *$ & 0,13 \\
\hline Epistemisches sit. Interesse (2) & - & 1,00 & $0,54 * * *$ & $0,34 * * *$ & $0,52 * * *$ & $0,23 * *$ \\
\hline Emotionales sit. Interesse (3) & - & - & 1,00 & $0,41 * * *$ & $0,31 * * *$ & $0,24 * *$ \\
\hline Wertbezogenes sit. Interesse (4) & - & - & - & 1,00 & $0,22 * *$ & $0,17 *$ \\
\hline Inhaltliche Relevanz (5) & - & - & - & - & 1,00 & 0,11 \\
\hline Wahrgenommene Authentizität der Laborgeräte (6) & _- & _- & _- & _- & _ & 1,00 \\
\hline
\end{tabular}

Signifikante Korrelationskoeffizienten sind hervorgehoben

${ }^{*} p<0,05 ; * * p<0,01 ; * * * p<0,001$ 
dell 2012). Solche wurden im Anschluss an die MANCOVA für die einzelnen $\mathrm{AV}$ mit den UV als Faktoren und den Kovariaten gerechnet. Zur Kontrolle des $\alpha$-Fehlers bei der Mehrfachtestung wurde das Signifikanzniveau für jede AV mit der Methode nach Benjamini und Hochberg (1995) auf dem 5\%-Niveau korrigiert. Mit dieser Methode wird im Vergleich zu anderen, wie der Bonferroni Korrektur, eine größere Teststärke erreicht (vgl. Thissen et al. 2002).

\section{Ergebnisse}

Die Mittelwerte und Standardabweichungen der abhängigen Variablen der vier Gruppen sind in Tab. 3 wiedergegeben. Eine Beschreibung befindet sich im Anhang.

\section{Zusammenhänge zwischen den Variablen}

Tab. 4 gibt einen Überblick über Zusammenhänge zwischen den Variablen. Die Korrelationskoeffizienten (Pearson's $r$ ) sind durchweg positiv. Das Sachinteresse an $\mathrm{Na}$ turwissenschaften korreliert signifikant mit der epistemischen $(r=0,49 ; p<0,001)$ und der emotionalen Komponente $(r=0,39 ; p<0,001)$ des situationalen Interesses sowie mit der wahrgenommenen inhaltlichen Relevanz $(r=0,50$; $p<0,001)$.

\section{Wirkungen der unabhängigen Variablen auf das situationale Interesse sowie die Wahrnehmung von inhaltlicher Relevanz und Authentizität der Laborgeräte}

Um die in Tab. 3 ersichtlichen Unterschiede zwischen den Versuchsgruppen in den drei Komponenten des situationalen Interesse, sowie der wahrgenommenen inhaltliche Relevanz und der wahrgenommenen Authentizität der Laborgeräte (abhängige Variablen) auf statistische Signifikanz zu prüfen, wurde eine multivariate Kovarianzanalyse durchgeführt, mit dem Lernort und den Laborgeräten als unabhängigen Variablen und dem Sachinteresse an $\mathrm{Na}$ turwissenschaften als Kovariate ${ }^{3}$. Das Gesamtmodell war statistisch signifikant $(F[4,143]=23,6 ; p<0,001)$. Die Haupteffekte der UV Lernort $(F[5,139]=3,034 ; p=0,012$; part. $\left.\eta^{2}=0,098\right)$ und Laborgeräte $(F[5,139]=16,114$; $p<0,001$; part. $\left.\eta^{2}=0,367\right)$ waren signifikant mit mittlerer bzw. großer Effektstärke. Die Kovariate Sachinteresse an Naturwissenschaften hatte ebenfalls einen signifikanten

\footnotetext{
${ }^{3}$ Das Geschlecht der Proband*innen hatte in der MANCOVA keinen statistisch bedeutsamen Einfluss $(F[5,138]=1,523$; $p=0,187)$, weshalb hier auf eine ausführliche Darstellung verzichtet wird. Details siehe Anhang.
}

Einfluss mit großer Effektstärke auf das Gesamtmodell $\left(F[5,139]=13,266 ; p<0,001\right.$; part. $\left.\eta^{2}=0,323\right)$.

In anschließenden univariaten ANCOVAs konnten weder in der emotionalen noch in der wertbezogenen Komponente des situationalen Interesses signifikante Unterschiede bezüglich der UV nachgewiesen werden. Allerdings wird in der emotionalen Komponente das globale Signifikanzniveau für den Faktor Laborgeräte nur knapp überschritten $\left(F[1,143]=3,751 ; p=0,055\right.$; part. $\left.\eta^{2}=0,026\right)$. Anders verhielt es sich bei der epistemischen Komponente: Diese war am authentischen Lernort Schülerlabor signifikant größer als in der Schule $(F[1,143]=5,310 ; p=0,023$; part. $\left.\eta^{2}=0,036\right)$. Auch die echten Laborgeräte $(F[1$, $143]=5,726 ; p=0,018$; part. $\left.\eta^{2}=0,038\right)$ sowie die Interaktion Lernort $*$ Laborgeräte $(F[1,143]=5,171 ; p=0,024$; part. $\eta^{2}=0,035$ ) erwiesen sich als statistisch bedeutsam für diese Interessenskomponente, jeweils mit kleiner Effektstärke. Die Kovariate Sachinteresse an Naturwissenschaften zeigte einen hoch signifikanten, großen Effekt auf das situationale epistemische Interesse $(F[1,143]=39,377$; $p<0,001$; part. $\eta^{2}=0,216$ ).

Für die wahrgenommene inhaltliche Relevanz erwies sich neben der Kovariate Sachinteresse an Naturwissenschaften mit großer Effektstärke $(F[1,143]=39,408$; $p<0,001$; part. $\left.\eta^{2}=0,216\right)$ nur der Lernort als statistisch bedeutsam (mittlerer Effekt, $F[1,143]=12,890 ; p<0,001$; part. $\eta^{2}=0,083$ ). Der authentische Lernort führte demnach zu einer höher ausgeprägten Relevanzwahrnehmung. Für die Laborgeräte galt dies hingegen nicht. Die wahrgenommene Authentizität der Laborgeräte wurde nur davon signifikant und mit großer Effektstärke beeinflusst, welche Laborgeräte (High-End oder Low-Cost) zum Einsatz kamen $\left(F[1,143]=75,614 ; p<0,001 ;\right.$ part. $\left.\eta^{2}=0,346\right)$. Die echten High-End-Laborgeräte wurden demnach unabhängig vom Lernort und ohne Einfluss der Kovariaten als deutlich authentischer wahrgenommen als die Low-CostGeräte.

\section{Diskussion}

Die hier beschriebene quasi-experimentelle Studie hatte zum Ziel, den Einfluss der Authentizität von Lernort und Laborgeräten auf das situationale Interesse und die Wahrnehmung von inhaltlicher Relevanz und Authentizität zu untersuchen. Die Forschungsfrage bezog sich auf Unterschiede zwischen den Versuchsgruppen mit unterschiedlich authentischen Lernsettings im situationalen Interesse sowie in der Wahrnehmung von inhaltlicher Relevanz und $\mathrm{Au}$ thentizität der Laborgeräte. Auf Basis früherer empirischer Studienergebnisse wurde angenommen, dass eine möglichst authentische Lernumgebung (Ort und Laborgeräte) die größten, positiven Effekte auf das Interesse sowie die 
Wahrnehmung von inhaltlicher Relevanz und Authentizität haben sollte (vgl. Pawek 2009; Lewalter und Geyer 2009; Betz 2018). Dies konnte im Wesentlichen bestätigt werden. Sowohl der authentische Lernort, als auch die authentischen Laborgeräte zeigten in der MANCOVA einen signifikanten Haupteffekt auf das Gesamtmodell. Dabei war auch das als Kovariate erhobene Sachinteresse an Naturwissenschaften statistisch bedeutsam.

Zwischen den Versuchsgruppen konnten bemerkenswerte Unterschiede in den einzelnen Interessenskomponenten festgestellt werden: Das situationale epistemische Interesse war in derjenigen Gruppe am stärksten ausgeprägt, die im Schülerlabor mit echten Laborgeräten arbeiten konnte. Für diese Interessenskomponente konnte also die erwartete Bedeutung der Authentizität bestätigt werden. Das authentischste Lernsetting konnte im Vergleich zu den weniger authentischen Alternativen das höchste situationale epistemische Interesse hervorrufen. Wie bei anderen Studien, zeigte sich zudem ein signifikanter positiver Zusammenhang zwischen dieser Interessenskomponente und dem Sachinteresse an Naturwissenschaften (vgl. Pawek 2009).

Epistemische Aspekte werden insbesondere mit einem sich entwickelnden individuellen Interesse in Verbindung gebracht (Mitchell 1993; Renninger und $\mathrm{Su}$ 2012). Dementsprechend sind höhere Werte im situationalen epistemischen Interesse auch ein Hinweis auf eine nachhaltigere Wirkung der Maßnahmen (vgl. Euler und Weßnigk 2011). Als Konsequenz bedeutet das für naturwissenschaftliche Schülerlabore, dass die gängige Praxis, forschungsnahe, authentische Laborgeräte einzusetzen mit Blick auf die Nachhaltigkeit der Maßnahmen gerechtfertigt erscheint. Das situationale epistemische Interesse wurde in einem forschungsnahen Schülerlabor mit echten, als authentisch empfundenen Laborgeräten stärker geweckt als mit vereinfachten Instrumenten. Die Frage, inwiefern der hohe Aufwand, der mit der Nutzung echter Laborgeräte in Schülerlaboren verbunden ist, gerechtfertigt ist, kann selbstredend nicht ausschließlich durch eine positive Wirkung auf das Interesse der Schüler*innen beantwortet werden. Sicherlich werden durch die Konfrontation mit echten Laborgeräten auch viele andere Ziele verfolgt und erreicht. Interesse wecken sollen sie jedoch auch (vgl. Euler und Schüttler 2020). Da solche Geräte meist sehr teuer und damit in Schulen kaum verfügbar sind, erscheint es zudem aus ökonomischer Sicht sinnvoll, authentische Instrumente aus der echten Forschung vor allem in Schülerlaboren einzusetzen und damit einer größeren Schülerzahl, als in einzelnen Schulen, zugänglich zu machen.

Für die emotionale und die wertbezogene Komponente des situationalen Interesses konnten hingegen zwischen den Versuchsgruppen keine signifikanten Unterschiede gefunden werden. Dies steht im Einklang mit der in aktuelleren Theorien zum Interesse stärker vorgenommene Trennung der emotionalen und wertbezogenen von der epistemischen Komponente. Mit Blick auf die wahrgenommene Interessantheit einer Situation werden von manchen Autoren sogar ausschließlich emotionale Aspekte betrachtet (Silvia 2008). Demnach wird eine Situation dann als interessant empfunden, wenn sie zugleich als neu und komplex, dabei aber auch als verständlich eingeschätzt wird. Die Lernsettings waren unabhängig von deren Authentizität gleichermaßen neu und komplex und die Verständlichkeit war durch die langjährige Erfahrung der Betreuer*innen mit den ausgiebig erprobten und optimierten Experimenten sichergestellt. Dies könnte erklären, warum die Lernsettings in den affektiven Komponenten des Interesses relativ ähnlich bewertet wurden. Die Nutzung der echten Laborgeräte führte zu einem etwas, aber nicht signifikant größeren emotionalen Interesse. Hier wurden ursprünglich signifikante Effekte erwartet, die sich aber statistisch nicht nachweisen ließen. Die erreichte Teststärke, basierend auf dem hier vorliegenden Stichprobenumfang und den tatsächlich gemessenen Werten, ist mit $1-\beta=0,49 \mathrm{zu}$ gering, um einen Effekt in dieser Variable nachzuweisen. Da die gemessenen Werte in der Skala des emotionalen situationalen Interesses, ebenso wie bei der wertbezogenen Komponente, zudem sehr nahe der oberen Skalengrenze lagen, sind jedoch auch Deckeneffekte wahrscheinlich (vgl. Döring und Bortz 2016).

Die wahrgenommene Authentizität der Laborgeräte wurde unabhängig vom Lernort nur von den verwendeten Messinstrumenten beeinflusst. Dies belegt, dass die Proband*innen die echten Laborgeräte als authentischer wahrnahmen als die einfacheren Instrumente. Wenn es also darum geht, aktuelle Forschung möglichst authentisch darzustellen, ist der Einsatz von Instrumenten, die so auch tatsächlich von Wissenschaftler*innen genutzt werden, gegenüber vereinfachten Materialien zu bevorzugen.

Die wahrgenommene inhaltliche Relevanz war unabhängig von den eingesetzten Laborgeräten in den Gruppen, die am authentischen Lernort arbeiten konnten, signifikant größer ausgeprägt. Eine Erklärung dafür könnte die Umgebung des Schülerlabors sein, welches sich an einer Großforschungseinrichtung mit ca. 2000 Mitarbeiter*innen befindet. Die Tatsache, dass so viele Menschen (deutlich wahrnehmbar) unter anderem an den Themen des Workshops forschen, unterstreicht deren Bedeutung. Auch bei dieser Variable zeigte sich ein signifikanter Zusammenhang mit dem Sachinteresse an Naturwissenschaften. Die wahrgenommene inhaltliche Relevanz korreliert zudem signifikant mit dem situationalen Interesse - insbesondere mit der epistemischen Komponente, was den theoretisch implizierten, engen Zusammenhang zwischen diesen Variablen empirisch weiter untermauert (vgl. auch Lewalter und Geyer 2009). 


\section{Limitationen und Ausblick}

An der Studie waren ausschließlich Gymnasiast*innen aus dem Großraum München beteiligt, was eine Übertragbarkeit auf andere Bildungszweige und andere Bundesländer erschwert. Zudem war der Stichprobenumfang mit 148 Proband*innen mit vollständigen Daten zwar vergleichbar mit ähnlichen Studien, dennoch wäre eine größere und heterogenere Stichprobe zu Gunsten der Generalisierbarkeit der Ergebnisse wünschenswert. Diese würde auch andere statistische Herangehensweisen (Teilgruppen- und Kausalanalysen) ermöglichen, welche beispielsweise auch differenziertere Einblicke in durchaus denkbare, hier aber nicht messbare Gendereffekte geben könnten. Diese standen nicht im Fokus der hier vorgestellten Untersuchung. Allerdings sollten Folgestudien mit entsprechendem Schwerpunkt Aufschluss darüber geben, inwiefern Gestaltungsmerkmale von Schülerlaboren Mädchen und Jungen unterschiedlich ansprechen.

Die Frage, warum die High-End-Laborgeräte im Schülerlabor mit Blick auf das situationale epistemische Interesse anders wirken als in der Schule, lässt sich auf Basis der erhobenen Daten nicht abschließend klären. Hierzu wären weitere Erhebungen, welche beispielsweise Konstrukte wie Neugier miteinbeziehen sollten, erforderlich. Die im Vergleich zu den anderen Gruppen stärkere Wirkung des authentischsten Lernsettings auf das situationale epistemische Interesse ist aus Sicht der Betreiber von forschungsnahen Schülerlaboren erfreulich, unterstreicht es doch die Bedeutung der Authentizität beim Schülerlaborbesuch. Kritisch anzumerken ist jedoch, dass es sich hierbei lediglich um eine Intention der Schüler*innen für ihre Zukunft handelt, deren tatsächlichen Auswirkungen auf das Lernverhalten der Jugendlichen nicht klar sind. In diesem Bereich existiert noch großer Forschungsbedarf - auch zu der Frage, wie Schülerlaborbesuche in den regulären Schulunterricht eingebettet werden können.

Der hier realisierte Workshop zur Infrarotfernerkundung konnte auch in den wenig authentischen Lernsettings ein vergleichsweise hohes situationales Interesse hervorrufen. Dabei wurden für den schulischen Unterricht relevante strahlungsphysikalische Aspekte behandelt. Der Kontext Infrarotfernerkundung eignet sich demnach gut für einen modernen Physikunterricht. Er ist jedoch wegen seiner großen Interdisziplinarität schwierig in den Schulalltag $\mathrm{zu}$ implementieren. Es wäre von großem Interesse zu untersuchen, inwiefern der Workshop auch von anderen Lehrkräften als den im DLR speziell ausgebildeten Personen durchgeführt und gewissermaßen aus dem Labor in die Schule übertragen werden könnte. Im Rahmen des vom DLR mit Mitteln des Bundesministeriums für Wirtschaft und Energie geförderten Projektes SatTec (www.sattec.org) wurden daher an Lehr- und Bildungsplänen orientierte Un- terrichtsmaterialien im Raumfahrtkontext entwickelt und in bundesweiten Lehrerfortbildungen vorgestellt. Eine Evaluation des so gestalteten Unterrichts, welcher durch andere Personen als die Autoren durchgeführt werden sollte, steht bislang noch aus.

Der auch in dieser Studie nachgewiesene enge Zusammenhang von wahrgenommener inhaltlicher Relevanz und insbesondere epistemischen Aspekten des situationalen Interesses ist von großer unterrichtspraktischer Bedeutung. Demnach ist für die Förderung eines länger anhaltenden Interesses an naturwissenschaftlichen Fächern auch die Frage zu klären, wie sich gerade die von den Schüler*innen wahrgenommene inhaltliche Relevanz, also letztlich die Erkenntnis, dass Naturwissenschaften einen wichtigen Beitrag für unser alltägliches Leben leisten, im Schulunterricht besser fördern lässt. Ein möglicher Weg besteht darin, im Schülerlabor behandelte Themen anschließend mit einfacheren „Do-It-Yourself“-Instrumenten - wie im beschriebenen Workshop - im Schulunterricht wieder aufzugreifen. Die Einbettung in den Schulunterricht kann nachweislich auch dem in manchen Studien zur Wirkung von Schülerlaborbesuchen berichteten Abfall des Interesses mit der Zeit entgegenwirken (vgl. Guderian 2007; Streller 2015; Pawek 2019). Zu diesem Zweck sind allerdings eine enge Zusammenarbeit und die Abstimmung zwischen den außerschulischen Lernorten, der Schule, lehrerbildenden Einrichtungen und nicht zuletzt auch den für die Weiterentwicklung der Bildungspläne verantwortlichen Akteuren erforderlich.

\section{Anhang}

\section{Deskriptive Beschreibung der Ergebnisse (Tab. 3)}

Die Werte für das Sachinteresse an Naturwissenschaften (T1) sind augenscheinlich nicht gleichverteilt und in den beiden Schülerlaborgruppen höher als bei den Gruppen in der Schule. Die Mittelwerte aller Komponenten des situationalen Interesses liegen in allen Treatmentgruppen über der Skalenmitte, in der emotionalen und der wertbezogenen Komponente deutlich. Auf deskriptiver Ebene fällt auf, dass die emotionale Komponente bei den Gruppen mit echten Laborgeräten etwas größer ausgeprägt ist, als bei denen mit vereinfachten Materialien. Die epistemische Komponente ist in der Gruppe, die mit echten Laborgeräten am authentischen Lernort arbeiten konnte, am größten. In den Schülerlaborgruppen ist zudem eine höher ausgeprägte wahrgenommene inhaltliche Relevanz zu beobachten, als in den Gruppen am wenig authentischen Lernort (Schule). Die wahrgenommene Authentizität der Laborgeräte ist für die echten Laborgeräte (High-End) deutlich höher ausgeprägt, als für die vereinfachten Geräte (Low-Cost). 


\section{Konfirmatorische Faktorenanalyse der eingesetzten Skala zum situationalen Interesse}

Die verwendete Skala zum situationalen Interesse wurde einer konfirmatorischen Faktorenanalyse unterzogen, um die Qualität des Messmodells zu kontrollieren. Dabei wurde das R-Paket lavaan genutzt (Döring und Bortz 2016). Von Multikollinearität ist bei den gemessenen Korrelationskoeffizienten nicht auszugehen (Tab. 4; $r<0,9$; vgl. Tabachnick und Fidell 2012).

Zur Beurteilung der Güte eines Messmodells werden in der Literatur verschiedene Prüfindizes genannt, die jedoch sensibel auf unterschiedliche Schwachpunkte des Modells sind (vgl. Weiber und Mühlhaus 2014). Daher wird empfohlen, mehrere verschiedene Gütekriterien zu überprüfen, welche unterschiedliche mögliche Schwächen des Modells aufdecken. Genannt werden inferenzstatistische Gütekriterien wie der Root-Mean-Square-Error (RMSEA), mit welchem kontrolliert wird, ob die Abweichungen der Werte des Messmodells zufallsbedingt sind. Nach Browne und Cudeck (1993, zitiert nach Weiber und Mühlhaus 2014) sind Werte von RMSEA $\leq 0,08$ akzeptabel. Absolute FitIndices wie der Quotient $\chi^{2} / \mathrm{df}$ und das Standardized Root Mean Square Residual (SRMR) zählen zu den deskriptiven Gütekriterien der Modellbeurteilung (vgl. auch Backhaus et al. 2015). Für sie werden in der Literatur als Grenzen für guten Modellfit $\chi^{2} / \mathrm{df} \leq 3$ bzw. SRMR $\leq 0,10$ genannt. Inkrementelle Fitmaße zum Modellvergleich überprüfen, ob das vorliegende Modell besser ist, als ein zufallsbedingtes Basismodell. Als Prüfgröße wird zumeist der Comparative Fit Index (CFI) genannt. Dabei kann von einer akzeptablen Modellanpassung ausgegangen werden, wenn $\mathrm{CFI} \geq 0,90$ gilt.

Die Modellpassung erwies sich im vorliegenden Fall als akzeptabel bis gut, wobei sich alle vier Kennwerte in den in der Literatur angegebenen Grenzen bewegen (Tab. 5). Zusammen mit den akzeptablen Werten für die interne Konsistenz der Skalen mit Cronbach's $\alpha$ von zumeist über 0,7 kann hier von einem insgesamt akzeptablen bis guten Messmodell ausgegangen werden.

\section{Betrachtung geschlechterspezifischer Unterschiede}

Die asymmetrische Geschlechterverteilung (vgl. Tab. 1) gab Anlass, das Geschlecht der Proband*innen als Variable in

Tab. 5 Kennwerte der konfirmatorischen Faktorenanalyse

\begin{tabular}{llll}
\hline$\chi^{2} / \mathrm{df}$ & CFI & RMSEA & SRMR \\
\hline 1,6 & 0,912 & 0,063 & 0,063 \\
\hline
\end{tabular}

Von einem akzeptablen bis guten Modellfit kann ausgegangen werden, wenn $\chi^{2} / \mathrm{df} \leq 3$; CFI $\geq 0,90$; RMSEA $\leq 0,08$ und SRMR $\leq 0,10$ gilt (Weiber und Mühlhaus 2014) die Datenauswertung miteinzubeziehen. Aus theoretischer Sicht sind hier Unterschiede denkbar. So ist beispielweise bekannt, dass sich Mädchen von Jungen in ihren Interessen an naturwissenschaftlichen Themen sowie in ihren Fachinteressen zum Teil stark unterscheiden (vgl. Holstermann und Bögeholz 2007; Krapp und Prenzel 2011; Reiss et al. 2016). Auf der anderen Seite berichten ähnliche Studien wie die vorliegende keine signifikanten oder nur vereinzelte und geringfügige Unterschiede zwischen Mädchen und Jungen im situationalen Interesse (vgl. Pawek 2009; Priemer et al. 2018). Raumfahrtthemen, wie im vorliegenden Workshop, werden zudem von beiden Geschlechtern als nahezu gleichermaßen ansprechend bewertet (Holstermann und Bögeholz 2007; Reiss et al. 2016; DeWitt und Bultitude 2018). Daher wurde bei der vorliegenden Studie davon ausgegangen, dass keine oder ggf. nur sehr kleine geschlechterspezifische Unterschiede bei der Wahrnehmung des Workshops in den abhängigen Variablen zu erwarten wären.

Diese Annahme wird durch die Ergebnisse gestützt. In einer Voranalyse der Daten mittels MANCOVA mit dem Geschlecht als Kovariate konnten keine signifikanten Gendereffekte nachgewiesen werden $(F[5,138]=1,523$; $p=0,187)$. Dies spiegelt sich auch in sehr ähnlichen Korrelationskoeffizienten aufgeschlüsselt nach Jungen und Mädchen wider (Tab. 6). In Tab. 7 sind die gemessenen Kennwerte der abhängigen Variablen und der Kovariate aufgeschlüsselt nach den Versuchsgruppen und dem Geschlecht wiedergegeben. In keiner der abhängigen Variablen sind signifikante Geschlechterunterschiede innerhalb der Versuchsgruppen festzustellen. Dies kann im Einklang mit oben genannten Studien als Hinweis darauf gewertet werden, dass das Thema des Workshops für beide Geschlechter gleichermaßen ansprechend war (vgl. Holstermann und Bögeholz 2007; Reiss et al. 2016; DeWitt und Bultitude 2018).

Die Ergebnisse belegen allerdings nicht, dass keine geschlechtsspezifischen Unterschieden in der Wahrnehmung der Proband*innen vorliegen. Wie Tab. 7 zeigt, sind im Sachinteresse an Naturwissenschaften durchaus Unterschiede zwischen den Geschlechtern innerhalb der Versuchsgruppen festzustellen, die jedoch aufgrund der kleinen Stichprobe größtenteils nicht signifikant sind. Eine erwähnenswerte Ausnahme bildet diejenige Gruppe, die im Schülerlabor mit den hochauthentischen High-EndLaborgeräten arbeiten konnte. Die Jungen in dieser Gruppe brachten im Vergleich zu den Mädchen ein im t-Test signifikant höheres Sachinteresse an Naturwissenschaften mit $(p=0,018$, korrigiert nach der Benjamini-HochbergMethode, vgl. Thissen et al. 2002). Diese eher ungünstigen Voraussetzungen der Mädchen konnten aber offenbar in gewissem Sinne kompensiert werden: Ihr situationales Interesse sowie die wahrgenommene inhaltliche Relevanz und Authentizität der Laborgeräte waren vergleichbar mit 
Tab. 6 Bivariate Korrelationen (Pearson's $r$ ) der abhängigen Variablen und der Kovariate Sachinteresse an Naturwissenschaften für Jungen (oberhalb) und Mädchen (unterhalb der Diagonalen) getrennt

\begin{tabular}{|c|c|c|c|c|c|c|}
\hline- & (1) & (2) & (3) & (4) & (5) & (6) \\
\hline Sachinteresse Naturwissenschaften (1) & 1,00 & $0,50 * * *$ & $0,37 * * *$ & 0,20 & $0,48 * * *$ & 0,09 \\
\hline Epistemisches sit. Interesse (2) & $0,44 * * *$ & 1,00 & $0,47 * * *$ & $0,34 * *$ & $0,54 * * *$ & 0,13 \\
\hline Emotionales sit. Interesse (3) & $0,47 * * *$ & $0,63 * * *$ & 1,00 & $0,42 * * *$ & $0,23 *$ & $0,31 * *$ \\
\hline Wertbezogenes sit. Interesse (4) & 0,08 & $0,41 * *$ & $0,42 * * *$ & 1,00 & $0,28 * *$ & 0,11 \\
\hline Inhaltliche Relevanz (5) & $0,50 * * *$ & $0,49 * * *$ & $0,40 * *$ & 0,16 & 1,00 & 0,12 \\
\hline $\begin{array}{l}\text { Wahrgenommene Authentizität der Laborgeräte } \\
\text { (6) }\end{array}$ & 0,19 & $0,39 * *$ & 0,14 & $0,30 *$ & 0,08 & 1,00 \\
\hline
\end{tabular}

Signifikante Korrelationskoeffizienten sind hervorgehoben

$* p<0,05 ; * * p<0,01 ; * * * p<0,001$

Tab. 7 Kennwerte der AV aufgeschlüsselt nach Geschlecht und Versuchsgruppen

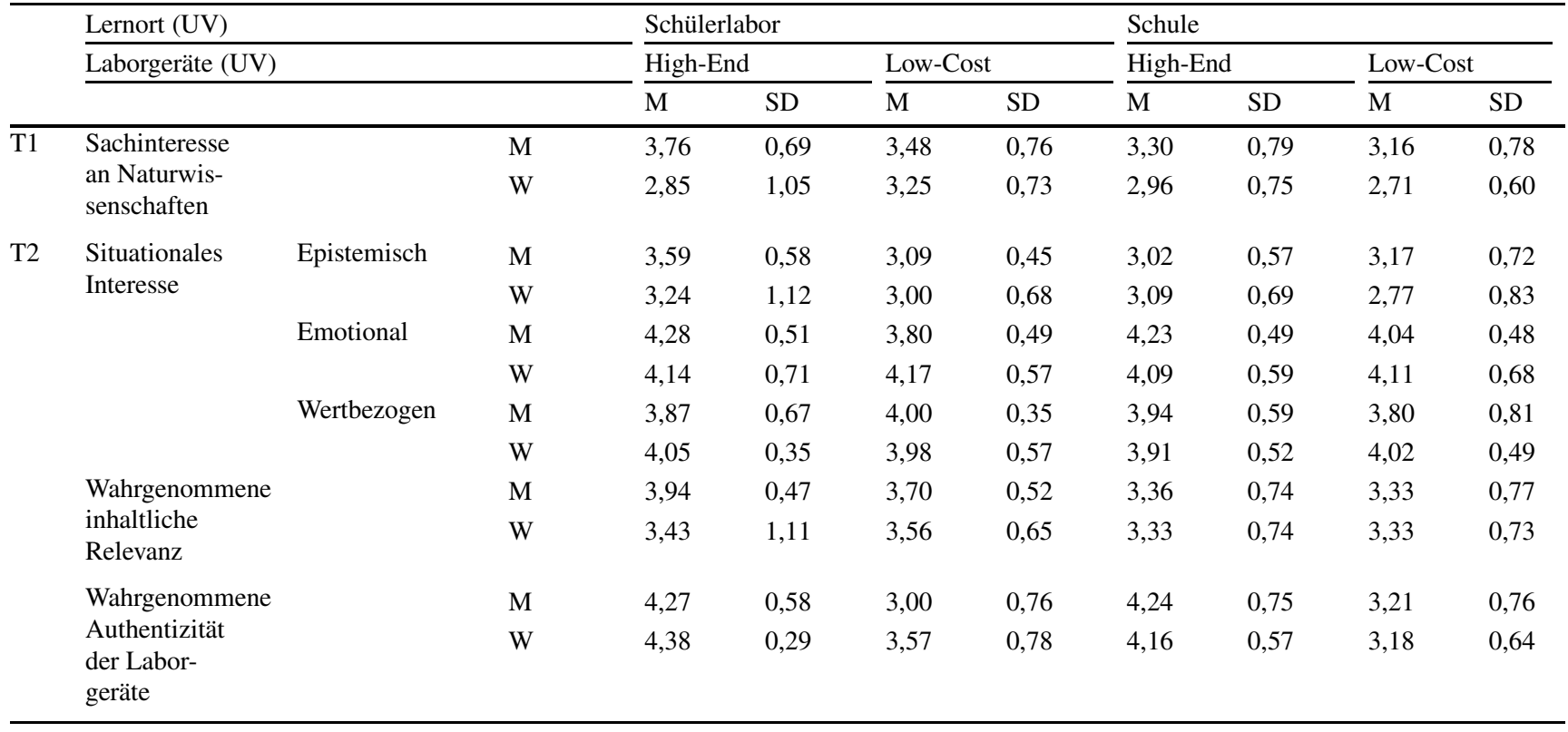

den Ergebnissen der Jungen sowie mit denen der anderen Gruppen. Dies kann als Hinweis dafür gedeutet werden, dass die besonders authentische Lernumgebung gerade die Mädchen und insbesondere solche mit einem eher schwach ausgeprägten Sachinteresse an Naturwissenschaften besonders anzusprechen vermochte ${ }^{4}$. Um diese Aspekte jedoch systematisch genauer zu beleuchten und statistisch abzusichern, wären eine deutlich größere Stichprobe und auch ein anderes Studiendesign mit anderen Forschungsfragen erforderlich gewesen.

Danksagung Für die Teilnahme an den Aktivitäten im Schülerlabor und in den Schulen gilt der besondere Dank den Schüler*innen sowie allen beteiligten Lehrkräften und den Schulleitungen für deren Unterstützung. Ein ganz besonderer Dank gilt dem bayerischen Staatsministerium für Unterricht und Kultus, welches diese Studie geneh-

\footnotetext{
${ }^{4}$ Weitergehende Analysen mittels univariater linearer Regressionen, auf die hier jedoch aus Gründen des Umfangs nicht eingegangen werden kann, stützen diese Vermutung.
}

migt und durch Abordnung des Autors an die Ludwig-MaximiliansUniversität München überhaupt erst ermöglicht hat. Dem Team des DLR_School_Lab Oberpfaffenhofen, insbesondere seinem langjährigen Leiter, Dr. Dieter Hausamann, sei herzlich für die produktive und motivierende Zusammenarbeit gedankt. Die Auswahl und Anwendung der statistischen Methoden wurde durch das Statistische Beratungslabor der LMU unterstützt.

Förderung Das Projekt SatTec, welches die vorliegende Arbeit unterstützt hat, wurde mit Mitteln des Bundes-Ministeriums für Wirtschaft und Energie durch das Deutsche Zentrum für Luft- und Raumfahrt e. V. (DLR) unter dem Förderkennzeichen 50RO1601 gefördert.

Funding Open Access funding enabled and organized by Projekt DEAL.

Open Access Dieser Artikel wird unter der Creative Commons Namensnennung 4.0 International Lizenz veröffentlicht, welche die Nutzung, Vervielfältigung, Bearbeitung, Verbreitung und Wiedergabe in jeglichem Medium und Format erlaubt, sofern Sie den/die ursprünglichen Autor(en) und die Quelle ordnungsgemäß nennen, einen Link zur Creative Commons Lizenz beifügen und angeben, ob Änderungen vorgenommen wurden. 
Die in diesem Artikel enthaltenen Bilder und sonstiges Drittmaterial unterliegen ebenfalls der genannten Creative Commons Lizenz, sofern sich aus der Abbildungslegende nichts anderes ergibt. Sofern das betreffende Material nicht unter der genannten Creative Commons Lizenz steht und die betreffende Handlung nicht nach gesetzlichen Vorschriften erlaubt ist, ist für die oben aufgeführten Weiterverwendungen des Materials die Einwilligung des jeweiligen Rechteinhabers einzuholen.

Weitere Details zur Lizenz entnehmen Sie bitte der Lizenzinformation auf http://creativecommons.org/licenses/by/4.0/deed.de.

\section{Literatur}

Alexander, P. A., \& Grossnickle, E. M. (2016). Positioning interest and curiosity within a model of academic development. In K. R. Wentzel \& D.B. Miele (Hrsg.), Handbook of motivation at school (2. Aufl. S. 188-208). New York: Routledge.

Anker-Hansen, J., \& Andreé, M. (2019). In pursuit of authenticity in science education. Nordic Studies in Science Education, 15(1), 54-66.

Backhaus, K., Erichson, B., \& Weiber, R. (2015). Fortgeschrittene multivariate Analysemethoden: eine anwendungsorientierte Einführung. Berlin, Heidelberg, New York: Springer.

Behrendt, H. (1990). Physikalische Schulversuche. Didaktische Theorie, methodische Praxis und die Einstellungen von Schülern zur Auswahl der Versuchsgeräte. (Dissertation) Pädagogische Hochschule Kiel, Kiel

Benjamini, Y., \& Hochberg, Y. (1995). Controlling the false discovery rate: a practical and powerful approach to multiple testing. Journal of the Royal Statistical Society, Series B, 57, 289-300.

Bennett, J., Lubben, F., \& Hogarth, S. (2007). Bringing science to life: A synthesis of the research evidence on the effects of contextbased and STS approaches to science teaching. Science education, 91(3), 347-370.

Betz, A. (2018). Der Einfluss der Lernumgebung auf die (wahrgenommene) Authentizität der linguistischen Wissenschaftsvermittlung und das Situationale Interesse von Lernenden. Unterrichtswissenschaft, 46(3), 261-278.

Betz, A., Flake, S., Mierwald, M., \& Vanderbeke, M. (2016). Modelling authenticity in teaching and learning contexts: a contribution to theory development and empirical investigation of the construct. In C. K. Looi, J. Polman, U. Cress \& P. Reimann (Hrsg.), Transforming learning, empowering earners: the International Conference of the Learning Sciences (ICLS) 2016(2) (S. 815-818). Singapore: International Society of the Learning Sciences.

Blankenburg, J., \& Scheersoi, A. (2018). Interesse und Interessenentwicklung. In D. Krüger, I. Parchmann \& H. Schecker (Hrsg.), Theorien in der naturwissenschaftsdidaktischen Forschung (S. 245-259). Berlin, Heidelberg, New York: Springer.

Bohnhardt, T., \& Schüttler, T. (2020). Fähigkeitsselbstkonzept im klassischen Schülerlabor. In K. Sommer, J. Wirth \& M. Vanderbeke (Hrsg.), Handbuch Forschen im Schülerlabor. (S. 111-118) Münster: Waxmann.

Brosius, H. B., Haas, A., \& Koschel, F. (2012). Methoden der empirischen Kommunikationsforschung (6. Aufl.). Wiesbaden: Springer.

Deci, E.L., \& Ryan, R.M. (2002). Overview of self-determination theory: An organismic dialectical perspective. In E.L. Deci \& R.M. Ryan (Hrsg.), Handbook of self-determination research (S. 3-33). Rochester: University of Rochester Press.

Demuth, R., Gräsel, C., Parchmann, I., \& Ralle, B. (Hrsg.). (2008). Chemie im Kontext - Von der Innovation zur nachhaltigen Verbreitung eines Unterrichtskonzepts. Münster, New York, München, Berlin: Waxmann.

DeWitt, J., \& Bultitude, K. (2018). Space science: the view from European school students. Research in Science Education, 50, 1-17.
Döring, N., \& Bortz, J. (2016). Forschungsmethoden und Evaluation. Wiesbaden: Springer.

Duit, R. (2010). Physik im Kontext: Konzepte, Ideen, Materialien für effizienten Physikunterricht; Kontextorientierung, neue LehrLernKultur, naturwissenschaftliche Denk- und Arbeitsweisen, moderne Physik und Technologie. Seelze: Friedrich.

Edelmann, W., \& Wittmann, S. (2012). Lernpsychologie (7. Aufl.). Weinheim: Beltz.

Engeln, K. (2004). Schülerlabors: Authentische und aktivierende Lernumgebungen als Möglichkeit, Interesse an Naturwissenschaften und Technik zu wecken. (Dissertation) Berlin: Logos

Euler, M., \& Schüttler, T. (2020). Schülerlabore. In E. Kircher, R. Girwidz \& H.E. Fischer (Hrsg.), Physikdidaktik: Methoden und Inhalte (S. 127-166). Berlin, Heidelberg, New York: Springer.

Euler, M., \& Weßnigk, S. (2011). Schülerlabore und die Förderung kreativer Potenziale. Plus Lucis, 19(1-2), 32-38.

Euler, M., Schüttler, T., \& Hausamann, D. (2015). Schülerlabore: Lernen durch Forschen und Entwickeln. In E. Kircher, R. Girwidz \& P. Häußler (Hrsg.), Physikdidaktik (S. 759-782). Berlin, Heidelberg, New York: Springer.

Fechner, S. (2009). Effects of context oriented learning on student interest and achievement in chemistry education. Studien zum Physik- und Chemielernen, Bd. 95. Berlin: Logos.

Frey, A., Taskinen, P., Schütte, K., Prenzel, M., Artelt, C., Baumert, J., Blum, W., Hammann, M., Klieme, E., \& Pekrun, R. (Hrsg.). (2009). PISA 2006 Skalenhandbuch. Dokumentation der Erhebungsinstrumente. Münster: Waxmann.

Gebhard, U., Höttecke, D., \& Rehm, M. (2017). Pädagogik der Naturwissenschaften: Ein Studienbuch. Wiesbaden: Springer.

Glowinski, I. (2007). Schülerlabore im Themenbereich Molekularbiologie als Interesse fördernde Lernumgebung. (Dissertation), Kiel: Christian-Albrechts-Universität

Guderian, P., Priemer, B., \& Schön, L.-H. (2006). In den Unterricht eingebundene Schülerlaborbesuche und deren Einfluss auf das aktuelle Interesse an Naturwissenschaften. Physik und Didaktik in Schule und Hochschule, 2(5), 142-149.

Guderian, P. (2007). Wirksamkeitsanalyse außerschulischer Lernorte - Der Einfluss mehrmaliger Besuche eines Schülerlabors auf die Entwicklung des Interesses an Physik. (Dissertation). HumboldtUniversität zu Berlin

Habig, S., van Vorst, H., \& Sumfleth, E. (2018). Merkmale kontextualisierter Lernaufgaben und ihre Wirkung auf das situationale Interesse und die Lernleistung von Schülerinnen und Schülern. Zeitschrift für Didaktik der Naturwissenschaften, 24(1), 99-114.

Haupt, O.J., Domjahn, J., Martin, U., Skiebe-Corrette, P., Vorst, S., Zehren, W., \& Hempelmann, R. (2013). Schülerlabor - Begriffsschärfung und Kategorisierung. $M N U, 66(6), 324-330$.

Hausamann, D. (2012). Extracurricular science labs for STEM talent support. Roeper Review, 34(3), 170-182.

Hidi, S., \& Renninger, K. A. (2006). The four-phase model of interest development. Educational psychologist, 41(2), 111-127.

Hidi, S., Renninger, K. A., \& Krapp, A. (2004). Interest, a motivational variable that combines affective and cognitive functioning. In D. Y. Dai \& R. J. Sternberg (Hrsg.), Motivation, emotion, and cognition: Integrative perspectives on intellectual functioning and development (S. 103-130). London: Routledge.

Holstermann, N., \& Bögeholz, S. (2007). Interesse von Jungen und Mädchen an naturwissenschaftlichen Themen am Ende der Sekundarstufe I. Zeitschrift für Didaktik der Naturwissenschaften, $13,71-86$.

Holstermann, N., Grube, D., \& Bögeholz, S. (2010). Hands-on activities and their influence on students' interest. Research in science education, 40(5), 743-757.

Höttecke, D., \& Rieß, F. (2015). Naturwissenschaftliches Experimentieren im Lichte der jüngeren Wissenschaftsforschung - Auf der Suche nach einem authentischen Experimentbegriff der Fachdidaktik. Zeitschrift für Didaktik der Naturwissenschaften, 21(1), 127-139. 
Itzek-Greulich, H., \& Vollmer, C. (2018). Quantitative Forschung zur Authentizität der Wissenschaftsvermittlung im Schülerlabor. Ein Diskussionsbeitrag. Unterrichtswissenschaft, 46(3), 321-326.

Itzek-Greulich, H., Flunger, B., Vollmer, C., Nagengast, B., Rehm, M., \& Trautwein, U. (2015). Effects of a science center outreach lab on school students' achievement-Are student lab visits needed when they teach what students can learn at school? Learning and Instruction, 38, 43-52.

Knogler, M., Harackiewicz, J.M., Gegenfurtner, A., \& Lewalter, D. (2015). How situational is situational interest? Investigating the longitudinal structure of situational interest. Contemporary Educational Psychology, 43, 39-50.

Köller, O., Baumert, J., \& Schnabel, K.U. (2000). Zum Zusammenspiel von schulischem Interesse und Lernen im Fach Mathematik: Längsschnittanalysen in den Sekundarstufen I und II. In U. Schiefele \& K. Wild (Hrsg.), Interesse und Lernmotivation - Untersuchungen zu Entwicklung, Förderung und Wirkung. Münster: Waxmann.

Krapp, A. (1992). Interesse, Lernen und Leistung. Neue Forschungsansätze in der Pädagogischen Psychologie. Zeitschrift für Pädagogik, 38(5), 747-770.

Krapp, A. (2000). Individuelle Interessen als Bedingung lebenslangen Lernens. In F. Achtenhagen \& W. Lempert (Hrsg.), Lebenslanges Lernen im Beruf - seine Grundlegung im Kindes- und Jugendalter (S. 54-75). Wiesbaden: Springer VS.

Krapp, A. (2002). Structural and dynamic aspects of interest development: Theoretical considerations from an ontogenetic perspective. Learning and instruction, 12(4), 383-409.

Krapp, A. (2005). Basic needs and the development of interest and intrinsic motivational orientations. Learning and Instruction, 15, 381-395.

Krapp, A., \& Prenzel, M. (2011). Research on interest in science: Theories, methods, and findings. International journal of science education, 33(1), 27-50.

Krüger, D., \& Vogt, H. (Hrsg.). (2007). Theorien in der biologiedidaktischen Forschung: Ein Handbuch für Lehramtsstudenten und Doktoranden. Berlin, Heidelberg, New York: Springer.

Kuckartz, U., Rädiker, S., Ebert, T., \& Schehl, J. (2013). Statistik. Eine verständliche Einführung. Wiesbaden: Springer.

Kuhn, J. (2010). Authentische Aufgaben im theoretischen Bereich von Instruktions- und Lehr-Lern-Forschung. Wiesbaden: Springer.

Lewalter, D., \& Geyer, C. (2009). Motivationale Aspekte von schulischen Besuchen in naturwissenschaftlich-technischen Museen. Zeitschrift für Erziehungswissenschaft, 12(1), 28-44.

Locherer, M., Hausamann, D., \& Schüttler, T. (2012). Practical science education in remote sensing at the DLR_School_Lab Oberpfaffenhofen. In Geoscience and Remote Sensing Symposium (IGARSS), 2012 IEEE International (S. 7389-7392).

Mertler, C. A., \& Reinhart, R.V. (2017). Advanced and multivariate statistical methods: Practical application and interpretation (6. Aufl.). New York: Taylor \& Francis.

Mierwald, M., Lehmann, T., \& Brauch, N. (2018). Zur Veränderung epistemologischer Überzeugungen im Schülerlabor: Authentizität von Lernmaterial als Chance der Entwicklung einer wissenschaftlich angemessenen Überzeugungshaltung im Fach Geschichte? Unterrichtswissenschaft, 46(3), 279-297.

Mitchell, M. (1993). Situational interest: Its multifaceted structure in the secondary school mathematics classroom. Journal of Educational Psychology, 85, 424-436.

Nachtigall, V., Rummel, N., \& Serova, K. (2018). Authentisch ist nicht gleich authentisch - Wie Schülerinnen und Schüler die Authentizität von Lernaktivitäten im Schülerlabor einschätzen. Unterrichtswissenschaft, 46(3), 299-319.

Palmer, D., Dixon, J., \& Archer, J. (2017). Using situational interest to enhance individual interest and science-related behaviours. $R e$ search in Science Education, 47(4), 731-753.

Parchmann, I., \& Kuhn, J. (2018). Lernen im Kontext. In D. Krüger, I. Parchmann \& H. Schecker (Hrsg.), Theorien in der naturwis- senschaftsdidaktischen Forschung (S. 193-207). Berlin, Heidelberg, New York: Springer.

Pawek, C. (2019). 20 Jahre Schülerlabore an Hochschulen und anderen Einrichtungen. Eine wissenschaftlich fundierte Erfolgsgeschichte. In C. Driesen \& A. Ittel (Hrsg.), Der Übergang in die Hochschule. Strategien, Organisationsstrukturen und Best Practices an deutschen Hochschulen (S. 143-157). Münster: Waxmann.

Pawek, C. (2009). Schülerlabore als interessefördernde außerschulische Lernumgebungen für Schülerinnen und Schüler aus der Mittel- und Oberstufe (Dissertation). Kiel: Christian-Albrechts Universität

Prenzel, M., \& Drechsel, B. (1996). Ein Jahr kaufmännische Erstausbildung: Veränderungen in Lernmotivation und Interesse. Unterrichtswissenschaft, 24(3), 217-234.

Priemer, B., \& Lewalter, D. (2009). Schülerlaborbesuche - eine Bereicherung für den naturwissenschaftlichen Unterricht. Praxis der Naturwissenschaften - Physik in der Schule, 4(58), 10-14.

Priemer, B., Menzl, C., Hagos, F., Musold, W., \& Schulz, J. (2018). Das situationale epistemische Interesse an physikalischen Themen von Mädchen und Jungen nach dem Besuch eines Schülerlabors. Zeitschrift für Didaktik der Naturwissenschaften, 24(1), 279-285

Reiss, K., Sälzer, C., Schiepe-Tiska, A., Klieme, E., \& Köller, O. (2016). PISA 2015 Eine Studie zwischen Kontinuität und Innovation. Münster: Waxmann.

Renninger, K. A., \& Hidi, S. (2011). Revisiting the conceptualization, measurement, and generation of interest. Educational Psychologist, 46(3), 168-184.

Renninger, K. A., \& Hidi, S. (2016). The power of interest for motivation and engagement. New York: Routledge.

Renninger, K. A., \& Su, S. (2012). Interest and its development. In R. M. Ryan (Hrsg.), The Oxford handbook of human motivation (S. 167-187). New York: Oxford University Press.

$\mathrm{R}$ Core Team (2020). R: A language and environment for statistical computing. R Foundation for Statistical Computing, Vienna, Austria. https://www.R-project.org/. Zugegriffen: 31.05.2021.

Scharfenberg, F.-J., \& Bogner, F. X. (2015). Empirische Analyse: Leistung zählt! In O. J. Haupt, et al. (Hrsg.), Schülerlabor-Atlas 2015. Schülerlabore im deutschsprachigen Raum (S. 24-31). Stuttgart: Klett MINT

Schiefele, U. (2009). Motivation. In E. Wild \& J. Möller (Hrsg.), Pädagogische Psychologie (S. 151-177). Berlin, Heidelberg, New York: Springer.

Schüttler, T., \& Girwidz, R. (2019). Kostengünstige Infrarotsensoren im Physikunterricht. Plus Lucis 2019(2), 16-21.

Schüttler, T., \& Zepp, S. (2019). Methoden der Infrarotfernerkundung im Physikunterricht. Plus Lucis, 2019(2), 22-27.

Schüttler, T., Girwidz, R., \& Zepp, S. (2017). Validierung von NDVIMessungen mit einer modifizierten Digitalkamera - Fernerkundung von Vegetation als Thema des Physikunterrichts. Physik und Didaktik in Schule und Hochschule PhyDid A 16(1), 81-90.

Schüttler, T., Maman, S., \& Girwidz, R. (2018). Physics teaching by infrared remote sensing of vegetation. Physics Education, 53(3).

Schüttler, T., Maman, S., \& Girwidz, R. (2019). Teaching remote sensing techniques with high-quality, low cost sensors. IEEE Geoscience and Remote Sensing Magazine, 185-190.

Seidel, T., Prenzel, M., \& Kobarg, M. (2005). How to run a video study. Technical report of the IPN video study. Münster: Waxmann.

Silvia, P. J. (2008). Interest-The curious emotion. Current directions in psychological science, 17(1), 57-60.

Simon, F. (2019). Der Einfluss von Betreuung und Betreuenden auf die Wirksamkeit von Schülerlaborbesuchen. (Dissertation) Dresden: Technische Universität

Streller, M. (2015). The educational effects of pre and post-work in outof-school laboratories. (Dissertation) Dresden: Technische Universität

Stuckey, M., Hofstein, A., Mamlok-Naaman, R., \& Eilks, I. (2013). The meaning of 'relevance' in science education and its impli- 
cations for the science curriculum. Studies in Science Education, $49(1), 1-34$.

Tabachnick, B. G., \& Fidell, L.S. (2012). Using multivariate statistics (6. Aufl.). Hoboken: Prentice Hall.

Tai, R. H., Qi Liu, C., Maltese, A. V., \& Fan, X. (2006). Career choice. Planning early for careers in science. Science, 312, 1143-1144.

Taskinen, P.H., Schütte, K., \& Prenzel, M. (2013). Adolescents' motivation to select an academic science-related career: The role of school factors, individual interest, and science self-concept. $E d u$ cational Research and Evaluation, 19, 717-733.

Thissen, D., Steinberg, L., \& Kuang, D. (2002). Quick and easy implementation of the Benjamini-Hochberg procedure for controlling the false positive rate in multiple comparisons. Journal of Educational and Behavioral Statistics, 27(1), 77-83.

van Vorst, H. (2013). Kontextmerkmale und ihr Einfluss auf das Schülerinteresse im Fach Chemie. Studien zum Physik- und Chemielernen, Bd. 145. Berlin: Logos. Dissertation van Vorst, H., Dorschu, A., Fechner, S., Kauertz, A., Krabbe, H., \& Sumfleth, E. (2015). Charakterisierung und Strukturierung von Kontexten im naturwissenschaftlichen Unterricht -Vorschlag einer theoretischen Modellierung. Zeitschrift für Didaktik der Naturwissenschaften, 21(1), 29-39.

Watzka, B., \& Girwidz, R. (2015). Einfluss der Kontextorientierung und des Präsentationsmodus von Aufgaben auf den Wissenserwerb und die Transferleistung physikalischer Inhalte. Zeitschrift für Didaktik der Naturwissenschaften, 21(1), 187-206.

Weiber, R., \& Mühlhaus, D. (2014). Strukturgleichungsmodellierung: Eine anwendungsorientierte Einführung in die Kausalanalyse mit Hilfe von AMOS, SmartPLS und SPSS (2. Aufl.). Berlin, Heidelberg, New York: Springer. 